Original Paper http://ajol.info/index.php/ijbcs http://indexmedicus.afro.who.int

\title{
Effets de la fertilisation organo-phosphatée sur la fertilité en matière organique et complexe adsorbant d'un ferralsol sous cacaoyers dans la région de Divo (Côte d'Ivoire)
}

\author{
Victor Tiéba OUATTARA ${ }^{1}$, Zoumana KONATE ${ }^{2}$, Gustave Francis MESSOUM ${ }^{3}$, \\ Emmanuel Koffi KASSIN ${ }^{4}$, Mathias Gnion TAHI ${ }^{4}$, Louis Anselme KOKO ${ }^{5}$, \\ Brahima $\mathrm{KONE}^{1}$, Emmanuel Acka $\mathrm{DICK}^{1}$ et Maméri CAMARA ${ }^{4 *}$ \\ 1Université Félix Houphouët Boigny (UFHB): 01 BPV34 Abidjan 01 (Côte d'Ivoire). \\ 2 Université Jean Lorougnon Guédé de Daloa / Unité de Formation et de Recherche en \\ Agroforesterie. BP 150 Daloa (Côte d'Ivoire). \\ 3 Direction Générale de la Recherche et de l'Innovation du Ministère de l'Enseignement Supérieur et de la \\ Recherche Scientifique. BP V151 Abidjan (Côte d'Ivoire). \\ 4 Centre National de Recherche Agronomique (CNRA) 01 BP 1740 Abidjan 01 (Côte d'Ivoire). \\ 5 Office Chérifien du Phosphate (OCP). \\ *Auteur correspondant ; E-mail : camara_mameri@yahoo.fr; (+225) $05256939 / 47504777$ / \\ 02021096
}

\section{RESUME}

Une étude a été conduite sur une période de deux ans, à la station de recherche sur le cacao du Centre National de Recherche Agronomique (CNRA) à Divo, en vue d'étudier les effets de la fertilisation organophosphatée sur la fertilité et la microvariabilité verticale de la matière organique, du complexe adsorbant et de quelques équilibres chimiques d'un ferralsol sous cacaoyers en replantation dans la région de Divo. Le dispositif expérimental était un bloc de Fisher à 4 répétitions avec 6 traitements : $\mathrm{T}_{0}$ (témoin), $\mathrm{T}_{1}$ (5t.ha ${ }^{-1}$ de compost), $\mathrm{T}_{2}\left(5\right.$ t.ha ${ }^{-1}$ compost $\left.+184 \mathrm{~kg} \cdot \mathrm{ha}^{-1} \mathrm{P}_{2} \mathrm{O}_{5}\right), \mathrm{T}_{3}\left(5\right.$ tha ${ }^{-1}$ compost $\left.+122,6 \mathrm{~kg} \cdot \mathrm{ha}^{-1} \mathrm{P}_{2} \mathrm{O}_{5}\right), \mathrm{T}_{4}\left(5 \mathrm{tha}^{-1}\right.$ compost $\left.+92 \mathrm{~kg} \cdot \mathrm{ha}^{-1} \mathrm{P}_{2} \mathrm{O}_{5}\right)$ et $\mathrm{T}_{5}\left(184 \mathrm{~kg} \cdot \mathrm{ha}^{-1} \mathrm{P}_{2} \mathrm{O}_{5}\right)$. La dynamique verticale du complexe adsorbant, de la matière organique et de quelques équilibres chimiques dans les sols sous cacaoyers ont été évaluées par les évolutions de ces paramètres en fonction des traitements et par horizons. Les résultats obtenus ont montré que la fertilisation mixte (organique et minérale) était meilleure que l'amendement organique ou la fertilisation minérale exclusive vis-à-vis du complexe, de la matière organique et des équilibres chimiques. Les traitements T2, T3, T4 et T1 ont amélioré les caractéristiques étudiées. Cependant, le traitement T2 s'est révélé meilleur pour l'amélioration des caractéristiques étudiées par rapport aux autres traitements (T1, T3, T4). La matière organique tout en se minéralisant a aussi permis l'accumulation des éléments nutritifs en surface surtout pour les régimes de fertilisation mixte, pool de l'absorption racinaire chez le cacaoyer. Cette étude a montré que la nutrition phosphatée des cacaoyers replantés peut être assurée convenablement avec une fertilisation organophosphatée à base de compost et d'une faible dose de TSP. Ces résultats pourraient être utilisés par les producteurs de cacao pour une replantation réussie durable, respectant l'environnement.

(C) 2018 International Formulae Group. All rights reserved.

Mots clés: Dynamique verticale, taux de redistribution, fumure organo-minérale, compost de résidus d'écabossage. 


\title{
Effects of organo-phosphate fertilization on fertility of organic matter and the adsorbent complex on ferralsol in cocoa trees in the region of Divo
}

\begin{abstract}
Fertilization is important for sustainable cocoa trees replantation in the context of climate change. A two-year study was conducted at the National Center for Agronomic Research (CNRA) Cocoa Research Station in Divo to study the effects of organophosphate fertilization on fertility and soil fertility vertical microvariability of the organic matter, the adsorbent complex and some chemical equilibria on ferralsol in cocoa trees replantation. The experimental design was a Fisher block with 4 replicates with 6 treatments: T0 (control), T1 (5 t.ha ${ }^{-1}$ compost), T2 (5t.ha ${ }^{-1}$ compost +184 kg.ha-1 P2O5), T3 (5t.ha ${ }^{-1}$ compost +122.6 kg.ha1) P2O5), T4 (5t.ha ${ }^{-1}$ compost +92 kg.ha-1 P2O5) and T5 (184 kg.ha-1 P2O5). The vertical dynamics of the adsorbent complex, organic matter and some chemical equilibria in cocoa soils have been evaluated by changes in these parameters as a function of treatments and horizons. The results obtained showed that mixed fertilization (organic and mineral) was better than organic amendment or mineral fertilization exclusive to the complex, organic matter and chemical equilibrium. T2, T3, T4 and T1 treatments improved the characteristics studied. However, T2 treatment was better for improving the characteristics studied compared to other treatments (T1, T3, T4). Organic matter while mineralizing also allowed the accumulation of nutrients at the surface especially for mixed fertilization regimes, pool of root absorption in cocoa trees. This study showed that the phosphate nutrition of replanted cocoa trees can be adequately ensured with organophosphate fertilization based on compost and a low dose of TSP. These results could be used by cocoa farmers for successful replanting that is sustainable, environmentally friendly.
\end{abstract}

(C) 2018 International Formulae Group. All rights reserved.

Keywords: Vertical dynamics, redistribution rate, organo-mineral fertilization, husk cocoa pod residues compost.

\section{INTRODUCTION}

Le contexte de la cacaoculture en Côte d'Ivoire est actuellement marqué par la disparition de la forêt qui était traditionnellement utilisée par les producteurs comme le précédent cultural idéal pour la culture du cacaoyer (Gockowski and Sonwa, 2010). Au même moment, les plantations sont majoritairement dans une phase de sénescence après plusieurs décennies d'exploitation (Aguilar et al., 2005), à laquelle s'ajoute une baisse de la fertilité des sols cultivés (Koko et al., 2009). Face à cette situation, les producteurs ont commencé à replanter sur des précédents non forestiers (vieilles plantations de cacaoyers ou de caféiers, jachères naturelles), mais avec des difficultés considérables (Assiri et al., 2015, Konaté et al., 2016) qui entraînent très souvent des échecs de replantation (Assiri, 2015). Dans ces sols, ce sont les principaux éléments nutritifs tels que $\mathrm{N}, \mathrm{P}, \mathrm{K}, \mathrm{Ca}$ et $\mathrm{Mg}$ qui sont épuisés après une longue exploitation des terres (Hartemink, 2005). Cette situation s'établit principalement non seulement en raison de la faible utilisation ou de l'inadéquation de l'application des engrais chimiques (Hartemink, 2005), mais aussi, des effets néfastes de son utilisation à long terme sur la fertilité des sols et sur l'environnement (Stadelmann et al., 2002). Des résidus d'écabossage qui sont disponibles et à la portée de la plupart des cacaoculteurs ont été recommandés (Koko, 2013) et pourraient servir d'alternatives pour améliorer, à moindre coût, la fertilité des sols. En effet, des expériences ont montré que l'apport de résidus d'écabossage améliore la teneur en potassium $(\mathrm{K})$, en magnésium $(\mathrm{Mg})$ et en calcium (Ca) dans le sol (Adejobi et al., 2014). Par ailleurs, d'autres auteurs ont également montré qu'une fertilisation intégrée (organique et inorganique) améliore considérablement, les teneurs en bases échangeables du sol ( $\mathrm{Ca}, \mathrm{Mg}$ et $\mathrm{K}), \mathrm{N}$ total et carbone organique $\mathrm{du}$ sol, en plus, les 
micronutriments tels que $\mathrm{Fe}, \mathrm{Cu}, \mathrm{Mn}$ et $\mathrm{Zn}$ (Sanni et al., 2015). Cette étude a été initiée afin de comprendre l'effet de l'utilisation des résidus de récoltes sous forme de compost dans les sols acides et leur dynamique en fertilisation des sols sous cacaoyers en Côte d'Ivoire.

\section{MATERIEL ET METHODES \\ Zone d'étude}

L'expérimentation a été conduite à la station de recherche du Centre National de Recherche Agronomique (CNRA) située à Divo dans le Centre-Ouest de la Côte d'Ivoire situé entre 100 et $200 \mathrm{~m}$ d'altitude avec les coordonnées de $05^{\circ} 46.198^{\prime}$ en latitude nord et $005^{0} 14.207^{\prime}$ en longitude ouest. La température moyenne annuelle est de l'ordre de 27 à $28{ }^{\circ} \mathrm{C}$ et la pluviométrie annuelle moyenne, de $1200 \mathrm{~mm}$. Les sols sont des Ferrasols fortement désaturés issus de granite (Kassin et al., 2009).

Le précédent cultural sur la parcelle d'étude était une jachère de 3 années à base d'une végétation de Chromolaena odorata qui s'est installée après abattage d'une vieille cacaoyère.

\section{Matériel \\ Matériel végétal}

Le matériel végétal était constitué de 7 hybrides de cacaoyers caractérisés par leur précocité de production. Les premières fleurs apparaissent à partir de 18 mois. Ils ont une bonne qualité des fèves et un bon rendement de l'ordre de 2 à 2,4 t/ha (Tableau 1). Les caractéristiques technologiques de ces hybrides de cacaoyer du CNRA énumérés par Kébé et al. (2009) sont présentés dans le Tableau 1.

\section{Matériel édaphique}

Le matériel édaphique est constitué d'échantillons de sols issus de la parcelle étudiée. Le pédopaysage est parsemé de quelques arbres de Gliricidia sepium avec les bananiers et constitué dans l'ensemble d'un microrelief plat avec une pente progressive de direction nord-ouest, rectiligne. La pente varie entre 5 et 10 p.c. Le sol de la parcelle profond de plus d'1,2 $\mathrm{m}$ est rouge- brun qui est un ferric acrisol selon N'Guessan et al., (2016). Elle est installée vers le mi-versant à environ $125 \mathrm{~m}$ du bas de versant (altitude 103 m). Les deux fosses ouvertes sont distantes de $50 \mathrm{~m}$ et sont sur la même séquence topographique. Les éléments grossiers de moins de 5 p.c se situent uniquement entre 40 et $60 \mathrm{~cm}$ (stone line) sont constitués à dominance de nodules ferromagnésiens d'environ 90 p.c. et d'un peu de quartz. C'est un sol gravillonnaire recouverte d'environ 40 $\mathrm{cm}$ de terre fine. La structure est grumeleuse en surface à plyèdrique à tendance nuciforme à miprofondeur et polyédrique subanguleuse en profondeur avec une texture sabloargileuse en surface, argilo-sableuse en miprofondeur et argileuse en profondeur. Un bon drainage jusqu'à $90 \mathrm{~cm}$ où on observe des horizons tachetés (tâches d'hydromorphie). On ne rencontre pas encore d'induration à $1,2 \mathrm{~m}$.

\section{Compost et fertilisants}

Le triple superphosphate (TSP) à 46 p.c. de $\mathrm{P}_{2} \mathrm{O}_{5}$ et un compost de résidus d'écabossage ont été utilisés comme matériel de fertilisation. Le compost a été obtenu à partir de résidus d'écabossage $(2 / 3)$ et de fiente de volaille (1/3), après 3 mois de compostage. Sa composition chimique en pourcentage (p.c.) après analyse a été la suivante : $\mathrm{N}=1, \mathrm{P}_{2} \mathrm{O}_{5}=1 ; \mathrm{K}_{2} \mathrm{O}=4 ; \mathrm{CaO}=$ 1,$7 ; \mathrm{MgO}=0,5$.

\section{Méthodes \\ Dispositif expérimental}

L'essai a été conduit selon un dispositif en blocs complets aléatoires, avec 6 traitements et 4 répétitions, sur une superficie de 0,9 ha. Les traitements ont été répartis de manière aléatoire dans chaque bloc de $102 \mathrm{~m}$ sur $10 \mathrm{~m}$. Ces blocs ont été disposés parallèlement et espacés de $10 \mathrm{~m}$ les uns des autres. Les parcelles élémentaires étaient constituées chacune de 30 cacaoyers espacés de $3 \mathrm{~m}$ sur 2,5 m (densité de 1333 plants/ha) (Figure 1). Des plants de bananiers, plantés 8 mois avant les cacaoyers, en interligne et à la 
même densité de1333/ha, ont servi d'ombrage temporaire.

\section{Traitements}

Dans chaque parcelle élémentaire, 30 cacaoyers ont été choisis pour recevoir les traitements suivants (Figure 1): $\mathrm{T}_{0}$ (témoin), $\mathrm{T}_{1}$ (5 t.ha ${ }^{-1}$ compost), $\mathrm{T}_{2}$ (5 t.ha ${ }^{-1}$ compost + 184 kg.ha $\left.{ }^{-1} \mathrm{P}_{2} \mathrm{O}_{5}\right), \mathrm{T}_{3}\left(5\right.$ t.ha $^{-1}$ compost + 122,6 kg.ha $\left.{ }^{-1} \mathrm{P}_{2} \mathrm{O}_{5}\right), \mathrm{T}_{4}\left(5 \mathrm{t} \cdot \mathrm{ha}^{-1}\right.$ compost +92 kg.ha $\left.{ }^{-1} \mathrm{P}_{2} \mathrm{O}_{5}\right)$ et $\mathrm{T}_{5}\left(184 \mathrm{~kg} \cdot \mathrm{ha}^{-1} \mathrm{P}_{2} \mathrm{O}_{5}\right)$ comme indiqué dans le Tableau 2.

Les apports ont été faits en surface chaque année aux pieds des cacaoyers, en couronne dans un rayon situé à $0,60 \mathrm{~m} \mathrm{du}$ collet des cacaoyers où les racines absorbantes sont les plus actives. L'application a été faite en deux périodes, l'une en début de la grande saison des pluies (avril) et l'autre, en début de la petite saison des pluies (septembre) pour chaque année.

\section{Echantillonnage de sols}

Les échantillons de sols ont été prélevés avec un tube cylindrique de sondage (Yoro, 2004). L'échantillonnage a été effectué sur les sols du précédent cultural (jachère de 3 ans) et de la cacaoyère 18 mois après plantation. Avant le prélèvement des sols, les débris végétaux et la litière en surface ont été systématiquement enlevés. Dans l'ensemble du précédent cultural et dans chaque parcelle élémentaire de l'essai, les sols des couches 0-5, 5-10, 10-15 et 15-20 $\mathrm{cm}$ ont été prélevés. Pour le précédent cultural, 10 carottes de sol ont été prélevées au hasard dans chaque couche puis regroupées et soigneusement mélangées afin de constituer les échantillons composites par couche, à analyser. Pour la parcelle d'essai, à la deuxième année, en août avant le prochain épandage, 10 carottes de sol ont été prélevées au hasard sous 10 cacaoyers dans chaque couche puis regroupées et soigneusement mélangées afin de constituer les échantillons composites par couche et par parcelle élémentaire. Ces échantillons ont été recomposés pour donner l'échantillon final par couche et par traitement, à analyser. $\mathrm{Au}$ total, 24 échantillons composites (4 couches x 6 traitements) ont été prélevés dans la parcelle d'essai. Les prélèvements ont été effectués dans la zone de fertilisation des cacaoyers (rayon de $0,6 \mathrm{~m}$ à partir du plant). Les échantillons de sol prélevés ont été séchés à l'air libre avant d'être tamisés à l'aide d'un tamis à mailles de $2 \mathrm{~mm}$ de diamètre et conservés dans des sachets plastiques pour les analyses au laboratoire.

\section{Analyses chimiques des échantillons de sols}

L'azote total a été analyse en deux étapes selon la méthode Kjeldahl dont la minéralisation et le dosage des ions ammonium (NH4) pour $1 \mathrm{~g}$ de terre fine. Le dosage du carbone organique a été fait par la méthode de Walkley-Black, basée sur l'oxydation du carbone organique par une solution normale de bichromate de potassium en excès $(\mathrm{K} 2 \mathrm{Cr} 2 \mathrm{O} 7)$ en présence d'acide sulfurique puis titré à l'aide du sel de Mohr $\left(\left[\mathrm{Fe}\left(\mathrm{SO}_{4}\right) 2(\mathrm{NH} 4)\right]\right.$. Les bases échangeables ou cations échangeables ont été extraits par la méthode à l'acétate d'ammonium à $\mathrm{pH} 7$. L'extraction a consisté en une prise de $5 \mathrm{~g}$ de sol tamisé à $2 \mathrm{~mm}$ dans un godet de $50 \mathrm{ml}$ avec $25 \mathrm{ml}$ d'une solution normale d'acétate d'ammonium $\mathrm{pH}$ selon la procédure décrite par Jackson, (1958) et adapté. A la suite de l'extraction des bases échangeables, le culot a été repris dans $75 \mathrm{ml}$ d'alcool éthylique pour être lavé.

Par la suite, le taux de la matière organique a été obtenu en multipliant le pourcentage de carbone par le coefficient 1,72 .

\section{Taux de redistribution verticale dans l'horizon 0-20 cm}

Elle a été déterminée par la relation suivante :

$\mathbf{P X i}=\left[\mathbf{X}_{\mathrm{i}} /\left(\mathbf{X}_{0-5}+\mathbf{X}_{5-10}+\mathbf{X}_{10-15}+\mathbf{X ~ 1}_{5-20}\right)\right]$ $\mathrm{x} 100$

PXi étant le pourcentage du minéral considéré pour chaque traitement dans un horizon donné ; i, l'horizon considéré, X la teneur de l'élément considéré des différents horizons étudiés $(0-5,5-10,10-15,15-20$ $\mathrm{cm})$.

\section{Analyses statistiques}

Les échantillons de sols ont été analysés sur la base de trois répétitions par traitement. Les 
données ont été soumises à une analyse de variance (ANOVA) à l'aide du logiciel GenStat Release 9.1. Le test de Newman et Keuls a été utilisé pour la comparaison des moyennes au seuil de 5 p.c. des variables étudiées ( $\mathrm{N}, \mathrm{C}, \mathrm{K}, \mathrm{Ca}$ et $\mathrm{Mg}$ du sol), de la $\mathrm{CEC}$, des équilibres entre ces teneurs) en fonction des traitements.

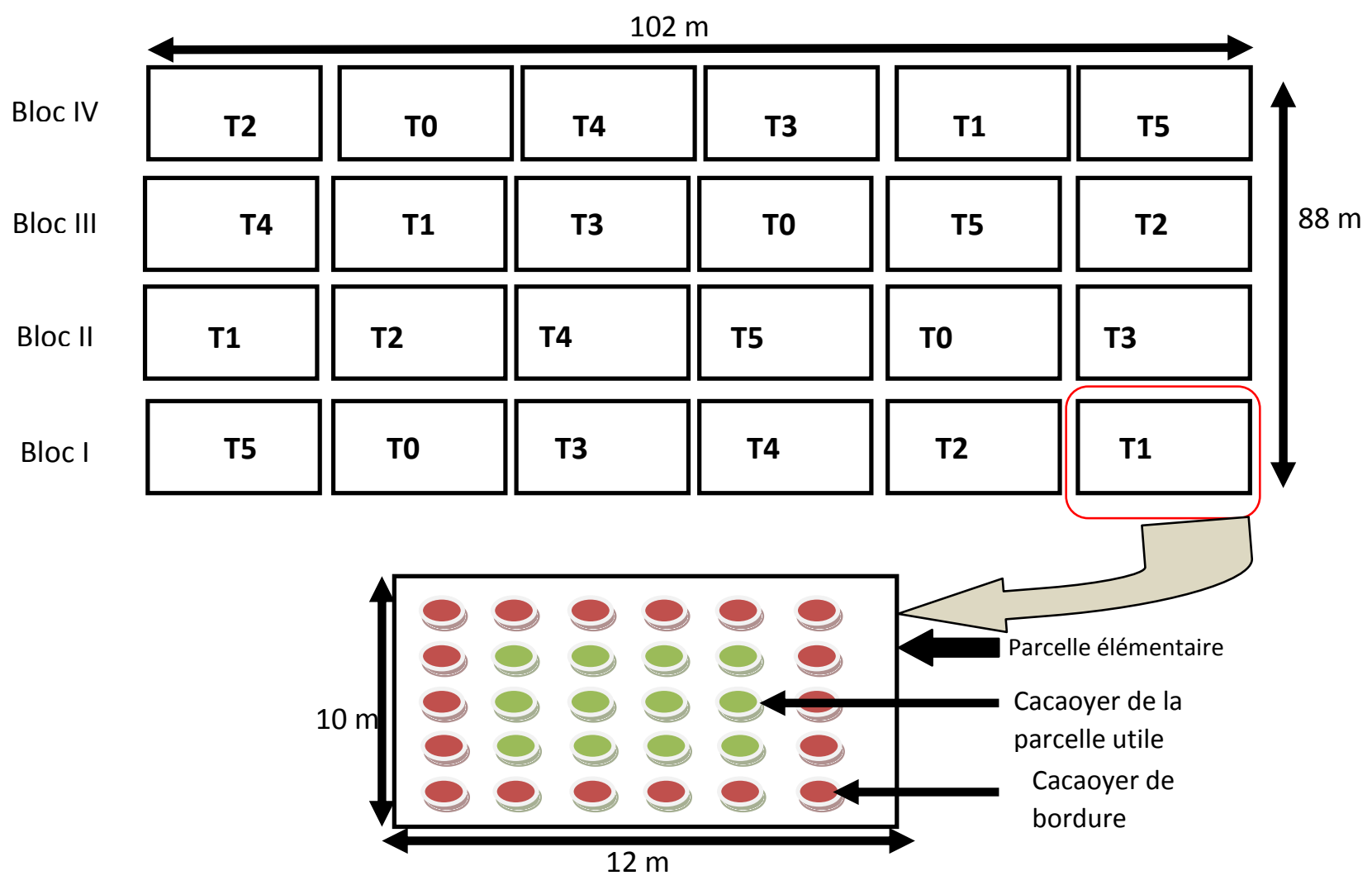

Figure 1 : Dispositif expérimental de l'étude.

Tableau 1 : Caractéristiques technologiques des hybrides de cacaoyers du CNRA.

\begin{tabular}{lcccc}
\hline Code & $\begin{array}{c}\text { Rendement } \\
\text { moyen (t/ha/an) }\end{array}$ & $\begin{array}{c}\text { Poids moyen de 100 fèves } \\
(\mathbf{g})\end{array}$ & $\begin{array}{c}\text { Taux moyen de matière } \\
\text { grasse (\% MS) }\end{array}$ & pH \\
\hline H109 & 2,2 & 120 & 57,0 & 5,4 \\
H125 & 2,0 & 107 & 58,5 & 5,4 \\
H117 & 2,1 & 117 & 58,0 & 5,6 \\
H140 & 2,0 & 113 & 58,7 & 5,5 \\
H153 & 2,1 & 130 & 54,4 & 5,4 \\
RB9 & 2,4 & 117 & 57,8 & 5,4 \\
H435 & 2,3 & 105 & 56,5 & 5,4 \\
\hline
\end{tabular}


Tableau 2: Différents régimes de fertilisation appliqués dans l'essai.

\begin{tabular}{|c|c|c|c|c|c|c|c|}
\hline \multirow[b]{2}{*}{ Traitements } & \multirow[b]{2}{*}{ Composition par ha } & \multicolumn{6}{|c|}{ Doses par fertilisant (kg/ha) } \\
\hline & & $\begin{array}{l}\mathrm{N} \mathrm{ha}^{-1} \text { apporté sous } \\
\text { forme de compost } \\
\text { (kg) }\end{array}$ & $\begin{array}{l}\mathrm{P}_{2} \mathrm{O}_{5} \mathrm{ha}^{-1} \text { sous } \\
\text { forme de TSP } \\
(\mathrm{kg})\end{array}$ & $\begin{array}{l}\mathrm{P}_{2} \mathrm{O}_{5} \mathrm{ha}^{-1} \text { sous } \\
\text { forme de } \\
\text { compost }(\mathrm{kg})\end{array}$ & $\begin{array}{l}\mathrm{K}_{2} \mathrm{O} \text { sous } \\
\text { forme de } \\
\text { compost }(\mathrm{kg})\end{array}$ & $\begin{array}{l}\mathrm{CaO} \text { sous forme } \\
\text { de compost }(\mathrm{kg})\end{array}$ & $\begin{array}{l}\text { MgO sous forme } \\
\text { de compost (kg) }\end{array}$ \\
\hline T0 & Sans apport & 0 & 0 & 0 & 0 & 0 & 0 \\
\hline $\mathrm{T} 1$ & 5 t compost.ha ${ }^{-1}$ & 52 & 0 & 52 & 208 & 88 & 26 \\
\hline $\mathrm{T} 2$ & $\mathrm{~T} 1+184 \mathrm{~kg} \mathrm{P}_{2} \mathrm{O}_{5} \mathrm{ha}^{-1}$ & 52 & 184 & 52 & 208 & 88 & 26 \\
\hline $\mathrm{T} 3$ & $\mathrm{~T} 1+122,6 \mathrm{~kg} \mathrm{P}_{2} \mathrm{O}_{5} \mathrm{ha}^{-1}$ & 52 & 122 & 52 & 208 & 88 & 26 \\
\hline $\mathrm{T} 4$ & $\mathrm{~T} 1+92 \mathrm{~kg} \mathrm{P}_{2} \mathrm{O}_{5} \mathrm{ha}^{-1}$ & 52 & 92 & 52 & 208 & 88 & 26 \\
\hline $\mathrm{T} 5$ & $184 \mathrm{~kg} \mathrm{P}_{2} \mathrm{O}_{5} \mathrm{ha}^{-1}$ & 0 & 184 & 0 & 0 & 0 & 0 \\
\hline
\end{tabular}




\section{RESULTATS \\ Evolution de la matière organique}

Le Tableau 3 montre les teneurs en $\mathrm{N}$ total, c-organique et la résultante en matière organique.

Les teneurs en $\mathrm{N}$ n'ont pas montré de différences significatives entre les traitements dans les horizons $(0-5,5-10,10-15$ et $15-20$ $\mathrm{cm})$.

Les différents traitements ont permis d'avoir des réponses variées des teneurs en carbone total des sols dans les horizons 0-5, 510 et $15-20 \mathrm{~cm}$. Dans l'ensemble des horizons, le traitement T2 a induit une teneur élevée alors que T0 et T5 ont engendré les teneurs les plus basses.

L'évolution du taux de matière organique a été similaire à celle du carbone organique avec un fort taux pour T2 par rapport aux autres traitements.

\section{Evolution du complexe adsorbant Teneur en calcium}

Dans les trois premières couches $(0-5$, 5-10, 10-15 cm), les teneurs les plus élevées ont été obtenues avec T1. Par contre, dans la couche $15-20 \mathrm{~cm}$, c'est T0 qui a induit la plus forte teneur des sols en calcium (Tableau 4).

\section{Teneur en magnésium}

Dans tous les horizons, les réponses des sols aux différents régimes de fertilisation ont varié. Les valeurs de $\mathrm{Mg}$ sont décroissantes de la surface à la profondeur (Tableau 4). Dans les horizons $0-5$ et $10-15$ $\mathrm{cm}, \mathrm{T} 1$ a permis d'avoir les teneurs les plus élevées, alors que dans les horizons 5-10 et 15-20 cm, c'est plutôt T2 qui a induit les teneurs les plus élevées. Le traitement T5, bien qu'étant faible au départ dans les horizons superficiels, a engendré une forte teneur dans l'horizon $15-20 \mathrm{~cm}$ avec 0,45 cmol. $\mathrm{kg}^{-1}$.

\section{Teneur en potassium}

Dans tous les horizons, les réponses des sols aux différents régimes de fertilisation ont été significativement différentes au seuil de 5 p.c. selon le test de SNK. Les moyennes comparées ont montré que dans tous les différents horizons de sols, les traitements T0 et T5 ont engendré les plus faibles teneurs en potassium. Cependant, les plus fortes teneurs ont été obtenues avec l'application des traitements T1, T2, T3 et T4 (Tableau 4).

\section{Teneur en sodium}

Dans tous les horizons de sols tees plus faibles teneurs en sodium ont été obtenues avec les traitements $\mathrm{T} 0$ et $\mathrm{T} 5$, alors que les plus fortes l'ont été avec T4 dans les horizons $(0-5,5-10$ et $10-15 \mathrm{~cm})$ et T3 dans les horizons (10-15 et $15-20 \mathrm{~cm}$ ). (Tableau 4).

\section{Capacité d'échange cationique (CEC)}

Les valeurs de CEC ont connu une augmentation après l'application des traitements $\mathrm{T} 2$ et $\mathrm{T} 1$ dans les couches $10-15$ et $15-20 \mathrm{~cm}$ et T3 dans la couche $10-15 \mathrm{~cm}$. La plus faible valeur de CEC dans les trois premières couches $(0-5,5-10,10-15 \mathrm{~cm})$ a été induite par l'application des traitements T5. T4 l'a été pour la dernière couche $(15-20 \mathrm{~cm})$. Les résultats obtenus ont également montré que pour la plupart des éléments nutritifs, les fortes teneurs en surface ont été induites par les traitements organo-minéraux et organiques seuls et les plus faibles pour les traitements T0 et T5. Dans les horizons sous-jacents (5-20 $\mathrm{cm})$, les teneurs les plus faibles ont été obtenues avec ces mêmes traitements (Tableau 4).

Evolution des équilibres chimiques dans les horizons en fonction des traitements C/N

Outre l'horizon 15-20 cm, les traitements appliqués n’ont pas influencé de manière significative le rapport $\mathrm{C} / \mathrm{N}$ dans les horizons 0-5, 5-10 et 10-15 cm.

Au niveau de l'horizon 15-20 cm, les traitements $\mathrm{T} 2$ et $\mathrm{T} 5$ avec respectivement, un rapport $\mathrm{C} / \mathrm{N}$ de 6,75 et 6,74 ont été les plus élevés (Tableaux 5 à 8).

\section{$(S B+6,15) / N$}

Les traitements appliqués ont influencé l'équilibre entre les bases échangeables et l'azote du sol dans les 4 horizons étudiés. En effet, quel que soit l'horizon, un maximum relatif a été obtenu avec l'application de $\mathrm{T} 1$. Cependant, dans l'horizon $5-10 \mathrm{~cm}$, en plus de 
T1, T2 a aussi permis d'avoir un rapport élevé avec 5,66 tandis que, dans l'horizon 15-20 $\mathrm{cm}$, c'est T0 qui l'a permis avec une valeur de 2,67. (Tableaux 5 à 8 ).

N/P

Les traitements ont affecté le rapport azote-phosphore dans le sol. Le maximum relatif a été obtenu avec T5 et le minimum relatif avec $\mathrm{T} 1$ pour les trois premiers horizons $(0-5,5-10$ et $10-15 \mathrm{~cm})$. Les traitements organo-minéraux ont induit des valeurs intermédiaires (Tableaux 5 à 8).

$N / K$

Les réponses ont été significatives en fonction des traitements dans les horizons. En effet, pour tous les horizons, le rapport N/P a été le plus élevé pour T0 et T5. Les faibles valeurs de rapports ont été engendrées par le traitement organique seul (T1) et ceux organominéraux (T2, T3 et T4) (Tableaux 5 à 8).

\section{K/CEC}

Les réponses du rapport K/CEC ont varié dans tous les horizons en fonction des régimes de fertilisation. Les rapports élevés ont été obtenus par les traitements T1, T2, T3 et T4 dans l'horizon $0-5 \mathrm{~cm}, \mathrm{~T} 3$ dans l'horizon 5-10 cm, T1, T2 et $\mathrm{T} 4$ dans l'horizon 10-15 $\mathrm{cm}$ et enfin $\mathrm{T} 4$ dans la dernière couche $(15-20 \mathrm{~cm})$. Les plus petits rapports l'ont été avec les traitements $\mathrm{T} 0$ et $\mathrm{T} 5$ (Tableaux 5 à 8).

\section{$\mathbf{M g} / \mathbf{K}$}

Le rapport $\mathrm{Mg} / \mathrm{K}$ a été surtout important dans les parcelles non fertilisées (T0) sur l'ensemble des 4 horizons et secondairement avec T5. Cependant, les traitements T1, T2, T3 et T4 ont engendré les plus faibles rapports (Tableaux 5 à 8).

\section{$\mathrm{Ca} / \mathrm{Mg}, \mathrm{Ca} / \mathrm{K}$ et $(\mathrm{Ca}+\mathrm{Mg}) / \mathrm{K}$}

Le traitement témoin a induit les valeurs les plus élevées des rapports $\mathbf{C a} / \mathbf{M g}$, $\mathbf{C a} / \mathbf{K}$ et $(\mathbf{C a}+\mathbf{M g}) / \mathbf{K}$ pour l'ensemble des quatre horizons. Les traitements organiques seuls et organo-minéraux ont engendré les plus faibles rapports (Tableaux 5 à 8 ).

\section{Pourcentages de Ca, $\mathrm{Mg}$ et $\mathrm{K}$}

Les traitements organo-minéraux et organiques seuls surtout dans le premier horizon $(0-5 \mathrm{~cm})$ et relativement dans l'horizon 5-10 cm ont engendré des équilibres les plus proches des optima (Tableaux 5 à 8 ).

Dynamique de la fertilité chimique dans les sols de chaque traitement Azote (N)

La teneur en azote total dans l'horizon $0-20 \mathrm{~cm}$ a été homogène avec l'application des traitements T0, T3, T4 et T5. En effet, elles ont varié de 0,11 à $0,22 \mathrm{~g} \cdot \mathrm{kg}^{-1}$ (T0), 0,11 à $0,29 \mathrm{~g} \cdot \mathrm{kg}^{-1}$ (T3), 0,09 à $0,29 \mathrm{~g} \cdot \mathrm{kg}^{-1}$ (T4) et de 0,08 à $0,18 \mathrm{~g} \cdot \mathrm{kg}^{-1}$ (T5). Par contre, avec l'application de $\mathrm{T} 1$ et $\mathrm{T} 2$, les teneurs ont été importantes dans les horizons $0-5 \mathrm{~cm}$ et 5-10 $\mathrm{cm}$ surtout pour T2 (Tableau 9).

\section{Carbone (C)}

Les teneurs en carbone total ont été élevées dans l'horizon $0-5 \mathrm{~cm}$ quel que soit le traitement et faible dans l'horizon $15-20 \mathrm{~cm}$ (Tableau 9).

\section{Matière organique (MO)}

Outre le traitement $\mathrm{T} 0$, où les horizons ont présenté des teneurs homogènes, tous les autres traitements ont permis une nette accumulation de la matière organique dans l'horizon de surface 0-5 cm (Tableau 9).

\section{$\mathrm{Ca}, \mathrm{Mg}, \mathrm{K}$ et CEC}

Pour tous les traitements appliqués, on a observé des teneurs en $\mathrm{Ca}, \mathrm{Mg}, \mathrm{K}$ et CEC élevées en surface $(0-5 \mathrm{~cm})$ et faible en profondeur dans la couche 15-20 cm (Tableau 9).

Les analyses statistiques ont révélé, pour la quasi-totalité des traitements, que les teneurs en éléments chimiques du sol ont subi une baisse de l'horizon de surface $0-5 \mathrm{~cm}$ à l'horizon de profondeur $15-20 \mathrm{~cm}$. Cependant, les teneurs en azote total $(\mathrm{N})$ et en sodium $\mathrm{Na}$ ont été relativement homogènes.

\section{Taux de redistribution verticale dans l'horizon 0-20 cm \\ Magnésium (Mg)}

Le magnésium a été préférentiellement réparti en surface $(0-5 \mathrm{~cm})$ pour une application du régime T1. Par ailleurs, T5 a engendré la plus faible proportion dans cet 
horizon. La répartition a été uniforme avec l'ensemble des traitements dans la couche 5$10 \mathrm{~cm}$. Dans les deux dernières couches, T5 a induit les plus fortes proportions tandis que les plus faibles proportions ont été obtenues avec T1 (Tableau 10).

\section{Calcium (Ca)}

Les teneurs en $\mathrm{Ca}$ ont été réparties en surface $(0-5 \mathrm{~cm})$ sans apport (T0) par rapport aux autres traitements avec 42 p.c. Par contre, la plus faible proportion dans cet horizon a été induite par T2 (31,64 p.c.). Dans l'horizon 5$10 \mathrm{~cm}$, la plus forte accumulation a été obtenue avec l'application de T2 (34,86 p.c.), les plus faibles ayant été permises par l'application de T0, T4 et T5. Dans la couche 10-15 cm, les plus fortes accumulations ont été induites par les traitements organominéraux (T2, T3 et T4) et minéral seul (T5) et les plus faibles avec T1 et T0. Dans la dernière couche $(15-20 \mathrm{~cm})$, seul $\mathrm{T} 1$ a permis une faible proportion avec 10,5 p.c. (Tableau $10)$.

\section{Potassium (K)}

Les deux premiers horizons (0-5 et 5$10 \mathrm{~cm}$ ) ont permis une accumulation homogène entre les traitements. Cependant, dans l'horizon 10-15 cm, la forte proportion a été obtenue avec T5 (21,19 p.c.) et les plus faibles proportions ont été obtenues avec $\mathrm{T} 2$ et T4. Par contre, la plus forte accumulation dans le dernier horizon $(15-20 \mathrm{~cm})$ a été induite par T0 (24,08 p.c.) et les plus faibles par T1, T2, T3 et T4 (Tableau 10).

\section{Sodium (Na)}

Les trois premiers horizons $(0-5,5-10$ et $15-15 \mathrm{~cm})$ ont présenté une répartition homogène quel que soit le traitement. Par contre, dans la dernière couche, la proportion la plus élevée a été obtenue avec T0 (29,01 p.c.) et la plus basse avec $\mathrm{T} 1$ pour une valeur de 14,53 p.c. (Tableau 10).

\section{Capacité d'Echange Cationique (CEC)}

Dans l'horizon 0-5 cm, seul T5 a induit une faible proportion de la capacité de charge cationique avec 26,8 p.c. Par contre, dans l'horizon 5-10 cm, la faible proportion l'a été par application de T3 avec 21,40 p.c. Dans la couche $10-15 \mathrm{~cm}$, l'accumulation a été plus hétérogène. Ainsi, les traitements T3 et T5 ont engendré une forte proportion des charges cationiques alors que les plus faibles ont été obtenues avec T2 (16,06 p.c.). Dans l'horizon $15-20 \mathrm{~cm}$, les proportions les plus élevées ont été observées avec l'application de régimes $\mathrm{T} 0$ et $\mathrm{T} 5$ et la plus faible taux de redistribution l'a été avec T4 avec 13,40 p.c. (Tableau 11). Azote (N)

Dans tous les horizons, la répartition en azote a été homogène pour l'ensemble des applications (Tableau 11).

\section{Carbone (C)}

Les trois premières couches $(0-5,5-10$ et $10-15 \mathrm{~cm}$ ) ont présenté des proportions homogènes avec l'ensemble des traitements. Cependant, dans la dernière couche (15-20 $\mathrm{cm})$, une forte proportion a été induite par T0 $(13,93)$ et une faible accumulation pour $\mathrm{T} 4$ avec une valeur de 8,75 p.c. (Tableau 11).

\section{Somme des Bases (SB)}

L'accumulation des bases échangeables dans l'horizon $0-5 \mathrm{~cm}$ a été élevée par application des traitements T0, T1 et $\mathrm{T} 4$ alors que les faibles répartitions ont été observées avec l'application des traitements T2, T3 et T5. Dans la couche 5-10 cm, T2 $(32,25$ p.c.) seul a induit une forte proportion. Par contre, dans l'horizon 10-15 cm, et 15-20 cm, T5 avec 20,60 p.c. a permis la meilleure répartition et $\mathrm{T} 1$ avec 19,17 p.c. la plus faible.

Pour la plupart des paramètres, T1, T2, $\mathrm{T} 3$ et $\mathrm{T} 4$ ont permis une faible répartition à partir de l'horizon 5-10 ou 10-15 cm et une meilleure répartition en surface et l'accumulation des éléments a été plus homogène pour T5 dans toutes les couches (Tableau 11). 
V. T. OUATTARA et al. /Int. J. Biol. Chem. Sci. 12(6): 2901-2921, 2018

Tableau 3 : Fertilité comparée de la matière organique par horizon en fonction des traitements.

\begin{tabular}{|c|c|c|c|c|c|c|c|c|c|c|c|c|}
\hline \multirow[t]{2}{*}{ Traitements } & \multicolumn{4}{|c|}{$\mathrm{C}\left(\mathrm{g} \cdot \mathrm{kg}^{-1}\right)$} & \multicolumn{4}{|c|}{$\mathbf{N}\left(\mathbf{g} \cdot \mathbf{k g}^{-1}\right)$} & \multicolumn{4}{|c|}{ MO $\left(\mathrm{g} \cdot \mathrm{kg}^{-1}\right)$} \\
\hline & $0-5 \mathrm{~cm}$ & $5-10 \mathrm{~cm}$ & $10-15 \mathrm{~cm}$ & $\begin{array}{c}15-20 \\
\mathrm{~cm}\end{array}$ & $0-5 \mathrm{~cm}$ & $5-10 \mathrm{~cm}$ & $\begin{array}{c}10-15 \\
\mathrm{~cm}\end{array}$ & $15-20 \mathrm{~cm}$ & $0-5 \mathrm{~cm}$ & $5-10 \mathrm{~cm}$ & $10-15 \mathrm{~cm}$ & $15-20 \mathrm{~cm}$ \\
\hline $\mathbf{T 0}$ & $1,55 \mathrm{~b}$ & $1,00 \mathrm{~b}$ & $0,74 \mathrm{a}$ & $0,5 \mathrm{~b}$ & $0,22 \mathrm{a}$ & $0,15 \mathrm{a}$ & $0,12 \mathrm{a}$ & $0,11 \mathrm{a}$ & $2,32 \mathrm{~d}$ & $1,67 \mathrm{~b}$ & $1,27 \mathrm{a}$ & $0,86 \mathrm{~b}$ \\
\hline $\mathrm{Tl}$ & $2,95 \mathrm{a}$ & $1,65 \mathrm{ab}$ & $1,10 \mathrm{a}$ & $0,66 a b$ & $0,32 \mathrm{a}$ & $0,21 \mathrm{a}$ & $0,16 \mathrm{a}$ & $0,13 \mathrm{a}$ & $5,07 \mathrm{ab}$ & $2,36 a b$ & 1,89 a & $1,13 a b$ \\
\hline T2 & $3,15 \mathrm{a}$ & $2,06 \mathrm{a}$ & $0,5 \mathrm{a}$ & $0,74 \mathrm{a}$ & $0,34 \mathrm{a}$ & $0,24 \mathrm{a}$ & $0,17 \mathrm{a}$ & $0,11 \mathrm{a}$ & $5,42 \mathrm{a}$ & $2,43 \mathrm{a}$ & $0,87 \mathrm{a}$ & $1,27 \mathrm{a}$ \\
\hline T3 & $2,95 \mathrm{a}$ & $1,31 \mathrm{ab}$ & $0,72 \mathrm{a}$ & $0,52 \mathrm{~b}$ & $0,29 \mathrm{a}$ & $0,17 \mathrm{a}$ & $0,12 \mathrm{a}$ & $0,11 \mathrm{a}$ & $5,07 \mathrm{ab}$ & $2,36 a b$ & $1,24 \mathrm{a}$ & $0,89 \mathrm{~b}$ \\
\hline T4 & $2,70 \mathrm{a}$ & $1,52 \mathrm{ab}$ & $0,72 \mathrm{a}$ & $0,44 \mathrm{~b}$ & $0,29 \mathrm{a}$ & $0,16 \mathrm{a}$ & $0,12 \mathrm{a}$ & $0,09 \mathrm{a}$ & $\begin{array}{c}4,64 \\
a b c\end{array}$ & $2,27 a b$ & $1,24 \mathrm{a}$ & $0,76 \mathrm{~b}$ \\
\hline T5 & $1,95 \mathrm{~b}$ & $1,25 \mathrm{ab}$ & $0,68 \mathrm{a}$ & $0,54 \mathrm{~b}$ & $0,18 \mathrm{a}$ & $0,13 \mathrm{a}$ & $0,11 \mathrm{a}$ & $0,08 \mathrm{a}$ & $3,28 \mathrm{bc}$ & $1,94 \mathrm{ab}$ & $1,17 \mathrm{a}$ & $0,93 \mathrm{~b}$ \\
\hline Moyenne & 2,53 & 1,47 & 0,74 & 0,57 & 0,27 & 0,18 & 0,13 & 0,11 & 4,30 & 2,52 & 1,28 & 0,97 \\
\hline $\mathrm{CV}(\%)$ & 3,1 & 6,1 & 10,8 & 2,9 & 6,00 & 3,4 & 2,1 & 1,1 & 5,6 & 6,8 & 13,4 & 3,6 \\
\hline Probabilité & $<0,001$ & 0,042 & 0,43 & 0,021 & 0,53 & 0,31 & 0,25 & 0,07 & 0,002 & 0,44 & 0,44 & 0,021 \\
\hline
\end{tabular}




\section{T. OUATTARA et al. /Int. J. Biol. Chem. Sci. 12(6): 2901-2921, 2018}

Tableau 4 : Fertilité comparée du complexe absorbant par horizon en fonction des traitements.

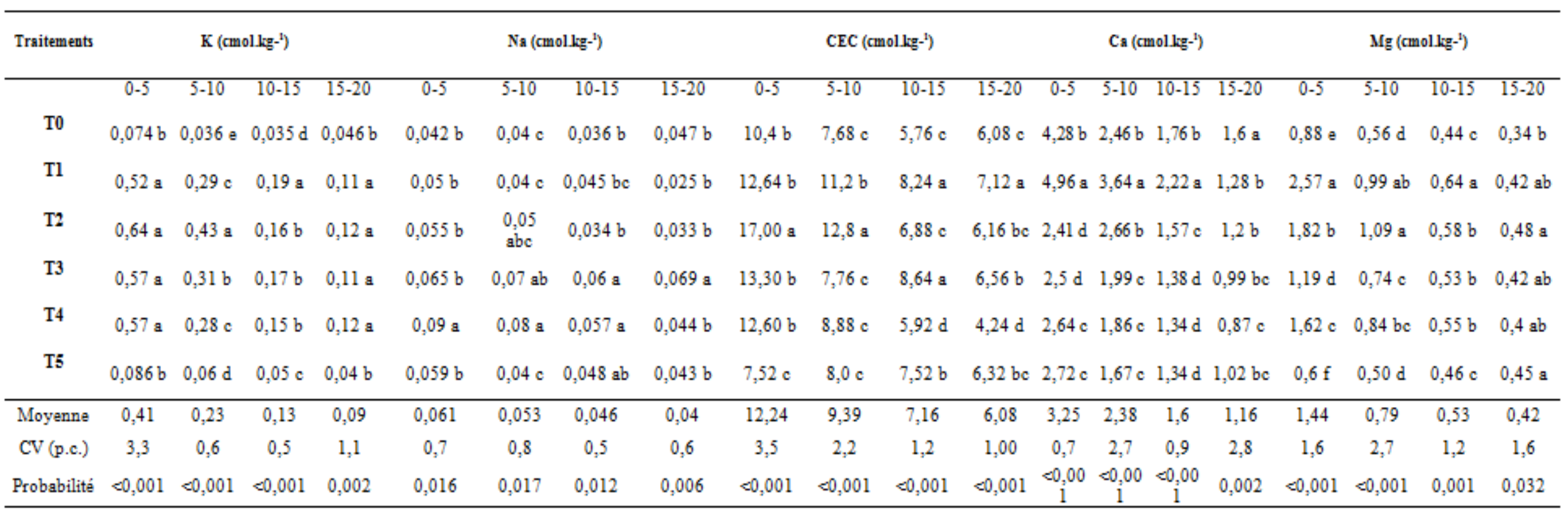


V. T. OUATTARA et al. /Int. J. Biol. Chem. Sci. 12(6): 2901-2921, 2018

Tableau 5 : Evolution des équilibres chimiques de l'horizon 0-5 cm.

\begin{tabular}{|c|c|c|c|c|c|c|c|c|c|c|c|c|c|}
\hline Traitements & $\mathrm{C} / \mathrm{N}$ & SB & $\begin{array}{c}\text { (SB+ } \\
6,15) / N\end{array}$ & N/P & $\mathbf{N} / \mathbf{K}$ & K/CEC & $\mathrm{Mg} / \mathrm{K}$ & $\mathrm{Ca} / \mathrm{Mg}$ & $\mathrm{Ca} / \mathrm{K}$ & $(\mathrm{Ca}+\mathrm{Mg}) / \mathrm{K}$ & $\mathrm{PCa}$ & pMg & pK \\
\hline T0 & $7,17 \mathbf{a}$ & $5,24 \mathrm{~b}$ & $6,59 \mathrm{bc}$ & 31,03 a & $3 \mathrm{a}$ & $0,71 \mathrm{c}$ & $11,94 \mathrm{a}$ & $4,87 \mathrm{a}$ & 58,04 a & 69,98 a & $81,78 \mathrm{a}$ & $16,81 \mathrm{~d}$ & $1,41 \mathrm{c}$ \\
\hline $\mathrm{T} 1$ & $9,24 \mathrm{a}$ & $8,04 a$ & $10,01 \mathrm{a}$ & 24,82 a & $0,61 \mathrm{~b}$ & $4,12 \mathrm{a}$ & $4,95 \mathrm{c}$ & $1,93 \mathrm{~b}$ & $9,54 \mathrm{c}$ & $14,49 \mathrm{c}$ & $61,6 \mathrm{~b}$ & $31,94 \mathrm{~b}$ & $6,46 \mathrm{~b}$ \\
\hline $\mathrm{T} 2$ & $9,34 \mathbf{a}$ & $4,88 \mathrm{c}$ & $6,97 \mathrm{~b}$ & $5,22 \mathrm{~b}$ & $0,53 \mathrm{c}$ & $3,77 \mathbf{a}$ & $2,85 \mathrm{~d}$ & $1,32 \mathrm{~d}$ & $3,78 \mathrm{~d}$ & $6,63 \mathrm{~d}$ & $49,49 \mathrm{~d}$ & $37,36 \mathbf{a}$ & $13,15 \mathrm{a}$ \\
\hline T3 & $11 \mathrm{a}$ & $4,26 \mathrm{c}$ & $6,04 \mathrm{bc}$ & $4,98 \mathrm{~b}$ & $0,5 \mathrm{c}$ & $4,28 \mathrm{a}$ & $2,1 \mathrm{~d}$ & $2,11 \mathrm{~b}$ & $4,44 \mathrm{~d}$ & $6,54 \mathrm{~d}$ & $58,69 \mathrm{~b}$ & $27,95 \mathrm{c}$ & $13,36 \mathrm{a}$ \\
\hline T4 & 10,07 a & $4,83 \mathrm{c}$ & $6,62 \mathrm{bc}$ & $5,05 \mathrm{~b}$ & $0,5 \mathrm{c}$ & $4,51 \mathrm{a}$ & $2,88 \mathrm{~d}$ & $1,62 \mathrm{c}$ & $4,69 \mathrm{~d}$ & $7,58 \mathrm{~d}$ & $54,6 \mathrm{c}$ & $33,61 \mathrm{~b}$ & $11,78 \mathrm{a}$ \\
\hline T5 & $12,91 \mathbf{a}$ & $3,4 \mathrm{~d}$ & $4,51 \mathrm{c}$ & $3,3 \mathrm{~b}$ & $2,17 \mathbf{a}$ & $1,14 \mathrm{~b}$ & $6,98 \mathrm{~b}$ & $4,56 \mathrm{a}$ & $31,78 \mathrm{~b}$ & $38,76 \mathrm{~b}$ & 79,93 a & $17,54 \mathrm{~d}$ & $2,52 \mathrm{c}$ \\
\hline Moyenne & 9,84 & 5,11 & 6,8 & 12,5 & 1,23 & 3,09 & 5,28 & 2,73 & 18,71 & 24 & 64,35 & 27,53 & 8,12 \\
\hline $\mathrm{CV}$ & 17,8 & 1,2 & 4,8 & 11,8 & 16,8 & 3,7 & 4,3 & 2 & 4,4 & 4,4 & 1,3 & 1,3 & 5,4 \\
\hline Probabilité & 0,78 & $<0,001$ & 0,004 & $<0,001$ & 0,02 & $=0,001$ & $\approx 0,001$ & $\approx 0,001$ & $<0,001$ & $=0,001$ & $<0,001$ & $\approx 0,001$ & $<0,001$ \\
\hline
\end{tabular}

Tableau 6: Evolution des équilibres chimiques de l'horizon $5-10 \mathrm{~cm}$.

\begin{tabular}{|c|c|c|c|c|c|c|c|c|c|c|c|c|c|}
\hline Traitements & $\mathrm{C} / \mathrm{N}$ & SB & $\begin{array}{c}\text { (SB+ } \\
6,15) / N\end{array}$ & N/P & $\mathbf{N} / \mathbf{K}$ & K/CEC & $\mathrm{Mg} / \mathrm{K}$ & $\mathrm{Ca} / \mathrm{Mg}$ & $\mathrm{Ca} / \mathrm{K}$ & $(\mathrm{Ca}+\mathrm{Mg}) / \mathrm{K}$ & $\mathrm{pCa}$ & $\mathrm{pMg}$ & $\mathbf{p K}$ \\
\hline T0 & $7,37 \mathbf{a}$ & $3,06 \mathrm{c}$ & $3,98 \mathrm{~b}$ & $24,96 \mathrm{~b}$ & $4,05 \mathrm{a}$ & $0,47 \mathrm{e}$ & $16,07 \mathrm{a}$ & 4,37 a & 70,9 a & 86,07 a & 80,42 a & $18,41 \mathrm{~d}$ & $1,17 \mathrm{~d}$ \\
\hline $\mathrm{T} 1$ & $7,87 \mathbf{a}$ & 4,92 a & $6,21 \mathrm{a}$ & 44,73 a & $0,73 \mathrm{c}$ & $2,57 \mathrm{c}$ & $3,44 \mathrm{c}$ & $3,7 \mathrm{~b}$ & $12,66 \mathrm{c}$ & $16,1 \mathrm{c}$ & $74,85 \mathrm{~b}$ & $20,1 \mathrm{~d}$ & $5,85 \mathrm{~b}$ \\
\hline $\mathrm{T} 2$ & $8,79 a$ & $4,18 \mathrm{~b}$ & $5,66 \mathrm{a}$ & $7,03 \mathrm{c}$ & $0,56 \mathrm{c}$ & $3,34 \mathrm{~b}$ & $2,5 \mathrm{c}$ & $2,43 \mathrm{c}$ & $6,24 \mathrm{c}$ & $8,8 \mathrm{c}$ & $63,57 \mathrm{c}$ & $26,18 \mathrm{ab}$ & $10,25 \mathrm{a}$ \\
\hline $\mathrm{T} 3$ & 7,81 a & $3,04 \mathrm{c}$ & $4,09 \mathrm{~b}$ & $5,77 \mathrm{c}$ & $0,54 \mathrm{c}$ & 4,05 a & $2,36 \mathrm{c}$ & $2,68 \mathrm{c}$ & $6,34 c$ & $8,7 \mathrm{c}$ & $65,32 \mathrm{~cd}$ & $24,37 \mathrm{bc}$ & $10,31 \mathrm{a}$ \\
\hline $\mathrm{T} 4$ & 9,42 a & $2,98 \mathrm{c}$ & $3,96 \mathrm{~b}$ & $10,81 \mathrm{c}$ & $0,56 \mathrm{c}$ & $3,19 \mathrm{~b}$ & $2,95 \mathrm{c}$ & $2,23 \mathrm{c}$ & $6,58 \mathrm{c}$ & $9,53 \mathrm{c}$ & $62,46 \mathrm{c}$ & $28,04 \mathrm{a}$ & 9,5 a \\
\hline T5 & 9,87 a & $2,23 \mathrm{~d}$ & $3,03 \mathrm{~b}$ & $3,45 \mathrm{c}$ & $2,21 \mathrm{~b}$ & $0,72 \mathrm{~d}$ & $8,86 \mathrm{~b}$ & $3,3 \mathrm{~b}$ & $29,28 \mathrm{~b}$ & $38,15 \mathrm{~b}$ & $74,76 \mathrm{~b}$ & $22,65 \mathrm{c}$ & $2,6 \mathrm{c}$ \\
\hline Moyenne & 8,52 & 3,4 & 4,49 & 16,13 & 1,44 & 2,39 & 6,04 & 3,12 & 21,85 & 27,89 & 70,09 & 23,29 & 6,61 \\
\hline $\mathrm{CV}$ & 9,6 & 2,8 & 4,7 & 12,1 & 8,4 & 2,5 & 9,1 & 2,3 & 9,7 & 9,6 & 0,6 & 1,7 & 3,7 \\
\hline Probabilité & 0,64 & $=0,001$ & 0,004 & $<0,001$ & $=0,001$ & $\approx 0,001$ & $\approx 0,001$ & $\approx 0,001$ & $\approx 0,001$ & $\approx 0,001$ & $<0,001$ & $\approx 0,001$ & $<0,001$ \\
\hline
\end{tabular}


V. T. OUATTARA et al. /Int. J. Biol. Chem. Sci. 12(6): 2901-2921, 2018

Tableau 7 : Evolution des équilibres chimiques de l'horizon 10-15 cm.

\begin{tabular}{|c|c|c|c|c|c|c|c|c|c|c|c|c|c|}
\hline Traitements & $\mathrm{C} / \mathrm{N}$ & SB & $\mathrm{SB}+6,15 \mathrm{~N}$ & N/P & $\mathrm{N} / \mathrm{K}$ & K/CEC & $\mathrm{Mg} / \mathrm{K}$ & $\mathrm{Ca} / \mathrm{Mg}$ & $\mathrm{Ca} / \mathrm{K}$ & $(\mathrm{Ca}+\mathrm{Mg}) / \mathrm{K}$ & $\mathrm{pCa}$ & $\mathrm{pMg}$ & $\mathrm{pK}$ \\
\hline T0 & $6,29 a$ & $2,23 \mathrm{~b}$ & $2,97 \mathrm{ab}$ & $100 \mathrm{~b}$ & 3,42 a & $0,61 \mathrm{c}$ & $12,67 \mathrm{a}$ & 4,02 a & 51,22 a & 63,88 a & 78,75 a & $19,68 \mathrm{~b}$ & $1,56 \mathrm{f}$ \\
\hline $\mathrm{T} 1$ & $6,9 \mathrm{a}$ & $3,05 \mathrm{a}$ & $4,04 \mathrm{a}$ & 134,29 a & $0,82 \mathrm{c}$ & $2,35 \mathrm{a}$ & $3,31 \mathrm{c}$ & $3,45 \mathrm{~b}$ & $11,43 \mathrm{c}$ & $14,74 \mathrm{c}$ & $72,61 \mathrm{~b}$ & $21,04 \mathrm{~b}$ & $6,36 \mathrm{~d}$ \\
\hline $\mathrm{T} 2$ & $5,88 \mathrm{a}$ & $2,31 \mathrm{~b}$ & $3,36 \mathrm{ab}$ & $26,07 \mathrm{c}$ & $1,03 \mathrm{c}$ & $2,4 \mathrm{a}$ & $3,5 \mathrm{c}$ & $2,72 \mathrm{c}$ & $9,53 \mathrm{c}$ & $13,03 \mathrm{c}$ & $67,94 \mathrm{c}$ & 24,94 a & $7,13 \mathrm{c}$ \\
\hline $\mathrm{T} 3$ & $6,14 \mathrm{a}$ & $2,09 \mathrm{c}$ & $2,83 \mathrm{ab}$ & $16,87 \mathrm{~d}$ & $0,69 \mathrm{c}$ & $2 \mathrm{~b}$ & $3,08 \mathrm{c}$ & $2,6 \mathrm{c}$ & $8,01 \mathrm{c}$ & $11,09 \mathrm{c}$ & $66,23 \mathrm{c}$ & $25,49 \mathrm{a}$ & 8,27 a \\
\hline $\mathrm{T} 4$ & $6,14 \mathrm{a}$ & $2,05 \mathrm{c}$ & $2,79 \mathrm{ab}$ & $34,07 \mathrm{c}$ & $0,77 \mathrm{c}$ & $2,62 \mathrm{a}$ & $3,56 \mathrm{c}$ & $2,44 \mathrm{c}$ & $8,67 \mathrm{c}$ & $12,23 \mathrm{c}$ & $65,55 \mathrm{c}$ & $26,89 \mathrm{a}$ & $7,56 \mathrm{ab}$ \\
\hline $\mathrm{T} 5$ & 6,21 a & $1,85 \mathrm{~d}$ & $2,53 \mathrm{c}$ & $6,91 \mathrm{e}$ & $2,2 \mathrm{~b}$ & $0,66 \mathrm{c}$ & $9,27 \mathrm{~b}$ & $2,93 \mathrm{c}$ & $27,07 \mathrm{~b}$ & $36,34 \mathrm{~b}$ & $72,52 \mathrm{~b}$ & 24,78 a & $2,69 \mathrm{e}$ \\
\hline Moyenne & 5,83 & 2,26 & 5,6 & 53,03 & 1,49 & 1,77 & 5,9 & 3,03 & 19,32 & 25,22 & 70,6 & 23,8 & 5,6 \\
\hline $\mathrm{CV}$ & 18,8 & 1 & 2,2 & 4,6 & 3,7 & 1,9 & 4,1 & 2,7 & 7,3 & 6,5 & 0,6 & 1,9 & 2,2 \\
\hline Probabilité & 0,46 & $<0,001$ & $<0,001$ & $<0,001$ & $<0,001$ & $<0,001$ & $<0,001$ & $<0,001$ & $<0,001$ & $<0,001$ & $<0,001$ & $<0,001$ & $<0,001$ \\
\hline
\end{tabular}

Tableau 8 : Evolution des équilibres chimiques de l'horizon 15-20 cm.

\begin{tabular}{|c|c|c|c|c|c|c|c|c|c|c|c|c|c|}
\hline Traitements & $\mathrm{C} / \mathrm{N}$ & SB & $\mathrm{SB}+6,15 \mathrm{~N}$ & $\mathrm{~N} / \mathrm{P}$ & $\mathrm{N} / \mathrm{K}$ & $\mathrm{K} / \mathrm{CEC}$ & $\mathrm{Mg} / \mathrm{K}$ & $\mathrm{Ca} / \mathrm{Mg}$ & $\mathrm{Ca} / \mathrm{K}$ & $(\mathrm{Ca}+\mathrm{Mg}) / \mathrm{K}$ & $\mathrm{pCa}$ & $\mathrm{pMg}$ & $\mathrm{pK}$ \\
\hline T0 & $4,54 \mathrm{~b}$ & $1,99 \mathrm{a}$ & $2,67 \mathrm{a}$ & $92,9 \mathrm{~b}$ & $2,39 \mathrm{a}$ & $0,76 \mathrm{c}$ & 7,49 a & 4,69 a & 34,92 a & 42,41 a & 80,47 a & $17,22 \mathrm{~d}$ & $2,31 \mathrm{c}$ \\
\hline $\mathrm{T} 1$ & $4,9 \mathrm{~b}$ & $1,8 \mathrm{ab}$ & $2,63 \mathrm{a}$ & $113 \mathrm{~b}$ & $1,23 \mathrm{c}$ & $1,53 \mathrm{~b}$ & $3,83 \mathrm{~b}$ & $3,06 \mathrm{~b}$ & $11,7 \mathrm{c}$ & $15,53 \mathrm{c}$ & $70,77 \mathrm{~b}$ & $23,18 \mathrm{c}$ & $6,05 \mathrm{~b}$ \\
\hline $\mathrm{T} 2$ & $6,75 \mathrm{a}$ & $1,8 \mathrm{ab}$ & $2,48 \mathrm{ab}$ & $61,9 \mathrm{c}$ & $0,92 \mathrm{~d}$ & $1,93 \mathrm{~b}$ & $4,03 \mathrm{~b}$ & $2,51 \mathrm{bc}$ & $10,08 \mathrm{~cd}$ & $14,1 \mathrm{c}$ & $66,67 \mathrm{bc}$ & $26,71 \mathrm{ab}$ & $6,62 \mathrm{ab}$ \\
\hline $\mathrm{T} 3$ & $4,75 \mathrm{~b}$ & $1,52 \mathrm{bc}$ & $2,19 \mathrm{ab}$ & 185,7 a & $0,99 \mathrm{~d}$ & $1,69 \mathrm{~b}$ & $3,84 \mathrm{~b}$ & $2,33 \mathrm{c}$ & $8,93 \mathrm{~d}$ & $12,77 \mathrm{c}$ & $64,88 \mathrm{c}$ & $27,82 \mathrm{ab}$ & $7,3 \mathrm{ab}$ \\
\hline $\mathrm{T} 4$ & $4,62 \mathrm{~b}$ & $1,39 \mathrm{c}$ & $1,97 \mathrm{~b}$ & $161,4 \mathrm{a}$ & $0,81 \mathrm{~d}$ & $2,83 \mathrm{a}$ & $3,39 \mathrm{~b}$ & $2,21 \mathrm{c}$ & $7,46 \mathrm{~d}$ & $10,85 \mathrm{c}$ & $62,9 \mathrm{c}$ & $28,51 \mathrm{ab}$ & $8,6 \mathrm{a}$ \\
\hline $\mathrm{T} 5$ & $6,74 \mathrm{a}$ & $1,51 \mathrm{bc}$ & $2 \mathrm{~b}$ & $8 \mathrm{~d}$ & $1,9 \mathrm{~b}$ & $0,66 \mathrm{c}$ & $10,68 \mathrm{a}$ & $2,29 \mathrm{c}$ & $24,27 \mathrm{~b}$ & $34,95 \mathrm{~b}$ & $67,51 \mathrm{bc}$ & 29,71 a & $2,78 \mathrm{c}$ \\
\hline Moyenne & 5,38 & 1,67 & 2,32 & 103,9 & 1,37 & 1,37 & 5,54 & 2,85 & 16,23 & 21,77 & 68,87 & 2,32 & 5,6 \\
\hline $\mathrm{CV}$ & 2,1 & 2,6 & 3,4 & 6,5 & 2,5 & 5,6 & 5,3 & 3,5 & 3,7 & 6,4 & 1,2 & 3,4 & 5,2 \\
\hline Probabilité & $<0,001$ & 0,011 & 0,034 & $<0,001$ & $<0,001$ & $<0,001$ & $<0,001$ & $<0,001$ & $<0,001$ & $<0,001$ & $<0,001$ & 0,034 & $<0,001$ \\
\hline
\end{tabular}


V. T. OUATTARA et al. / Int. J. Biol. Chem. Sci. 12(6): 2901-2921, 2018

Tableau 9 : Dynamique de la fertilité chimique en fonction des horizons pour chaque traitement.

\begin{tabular}{|c|c|c|c|c|c|c|c|c|c|}
\hline Traitements & $\begin{array}{c}\text { Horizons } \\
\text { (cm) }\end{array}$ & $\mathrm{N}\left(\mathrm{g} \cdot \mathrm{kg}^{-1}\right)$ & $\mathrm{C}\left(\mathrm{g} \cdot \mathrm{kg}^{-1}\right)$ & M0 (g. $\left.\mathrm{kg}^{-1}\right)$ & $\mathrm{Ca}\left(\mathrm{cmol} \mathrm{kg}^{-1}\right)$ & $\mathrm{Mg}\left(\mathrm{cmol} \mathrm{kg}-{ }^{\mathrm{l}}\right)$ & $\mathrm{K}\left(\mathrm{cmol} \mathrm{kg}_{-}{ }^{-1}\right)$ & $\mathrm{Na}\left(\mathrm{cmol} \mathrm{kg}_{-}{ }^{-1}\right)$ & CEC $\left(\mathrm{cmol} \mathrm{kg}_{-}{ }^{1}\right)$ \\
\hline \multirow{4}{*}{ T0 } & $0-5$ & $0,22 \mathrm{a}$ & $1,55 \mathrm{a}$ & $2,32 \mathbf{a}$ & $4,29 \mathrm{a}$ & $0,88 \mathrm{a}$ & $0,07 \mathbf{a}$ & $0,04 \mathrm{a}$ & 10,4 a \\
\hline & $5-10$ & $0,15 \mathbf{a}$ & $1 \mathrm{~b}$ & $1,72 \mathbf{a}$ & $2,46 \mathrm{~b}$ & $0,56 \mathrm{~b}$ & $0,04 \mathrm{~b}$ & $0,037 \mathbf{a}$ & $7,68 \mathrm{~b}$ \\
\hline & $10-15$ & $0,12 \mathrm{a}$ & $0,74 \mathrm{bc}$ & $1,27 \mathbf{a}$ & $1,76 \mathrm{c}$ & $0,44 \mathrm{c}$ & $0,03 \mathrm{~b}$ & $0,036 \mathrm{a}$ & $5,76 \mathrm{c}$ \\
\hline & $15-20$ & $0,11 \mathrm{a}$ & $0,5 \mathrm{c}$ & $0,86 \mathrm{a}$ & $1,6 \mathrm{c}$ & $0,34 \mathrm{~d}$ & $0,05 \mathrm{~b}$ & $0,047 \mathbf{a}$ & $6,08 \mathrm{c}$ \\
\hline \multirow{4}{*}{$\mathrm{T} 1$} & $0-5$ & $0,32 \mathrm{a}$ & $2,95 \mathrm{a}$ & $5,07 \mathbf{a}$ & $4,96 \mathrm{a}$ & $2,57 \mathbf{a}$ & $0,52 \mathrm{a}$ & $0,057 \mathbf{a}$ & $12,64 \mathrm{a}$ \\
\hline & $5-10$ & $0,21 \mathrm{~b}$ & $1,65 \mathrm{~b}$ & $2,84 \mathrm{~b}$ & $3,64 \mathrm{~b}$ & $0,99 \mathrm{~b}$ & $0,29 \mathrm{~b}$ & $0,045 \mathrm{a}$ & $11,2 \mathrm{a}$ \\
\hline & $10-15$ & $0,16 \mathrm{~b}$ & $1,1 \mathrm{c}$ & $1,89 \mathrm{c}$ & $2,21 \mathrm{c}$ & $0,64 \mathrm{c}$ & $0,19 \mathrm{c}$ & 0,045 a & $8,24 b$ \\
\hline & $15-20 \mathrm{c}$ & $0,13 \mathrm{~b}$ & $0,66 \mathrm{~d}$ & $1,3 \mathrm{~d}$ & $1,28 \mathrm{~d}$ & $0,42 \mathrm{~d}$ & $0,11 \mathrm{~d}$ & $0,025 \mathrm{a}$ & $7,12 \mathrm{~b}$ \\
\hline \multirow{4}{*}{$\mathrm{T} 2$} & $0-5$ & $0,34 \mathbf{a}$ & $3,15 \mathbf{a}$ & $5,42 \mathrm{a}$ & $2,41 \mathbf{a}$ & $1,82 \mathrm{a}$ & $0,64 \mathbf{a}$ & $0,05 \mathbf{a}$ & $17 \mathbf{a}$ \\
\hline & $5-10$ & $0,24 \mathrm{ab}$ & $2,06 \mathrm{a}$ & $3,54 \mathrm{a}$ & $2,66 \mathrm{a}$ & $1,09 \mathrm{~b}$ & $0,43 \mathrm{~b}$ & $0,05 \mathbf{a}$ & $12,8 \mathrm{~b}$ \\
\hline & $10-15$ & $0,17 \mathrm{c}$ & $0,5 \mathrm{~b}$ & $0,87 \mathrm{~b}$ & $1,57 \mathrm{~b}$ & $0,58 \mathrm{c}$ & $0,16 \mathrm{c}$ & $0,03 \mathrm{~b}$ & $6,88 \mathrm{c}$ \\
\hline & $15-20$ & $0,11 \mathrm{c}$ & $0,74 \mathrm{~b}$ & $1,27 \mathrm{~b}$ & $1,2 \mathrm{~b}$ & $0,48 \mathrm{c}$ & $0,12 \mathrm{c}$ & $0,03 \mathrm{~b}$ & $6,16 \mathrm{c}$ \\
\hline \multirow{4}{*}{ T3 } & $0-5$ & $0,29 \mathrm{a}$ & $2,95 \mathrm{a}$ & $5,07 \mathbf{a}$ & $2,5 \mathrm{a}$ & $1,19 \mathrm{a}$ & $0,57 \mathbf{a}$ & $0,06 \mathrm{a}$ & $13,3 \mathbf{a}$ \\
\hline & $5-10$ & $0,17 \mathbf{a}$ & $1,31 \mathrm{~b}$ & $2,25 \mathrm{~b}$ & $1,99 \mathrm{~b}$ & $0,74 \mathrm{~b}$ & $0,31 \mathrm{~b}$ & $0,07 \mathbf{a}$ & $7,76 \mathrm{c}$ \\
\hline & $10-15$ & $0,12 \mathrm{a}$ & $0,72 \mathrm{c}$ & $1,24 \mathrm{c}$ & $1,38 \mathrm{c}$ & $0,55 \mathrm{c}$ & $0,17 \mathrm{c}$ & $0,06 \mathrm{a}$ & $8,64 \mathrm{~b}$ \\
\hline & $15-20$ & $0,11 \mathbf{a}$ & $0,52 \mathrm{~d}$ & $0,89 \mathrm{~d}$ & $0,98 \mathrm{~d}$ & $0,42 \mathrm{c}$ & $0,11 \mathrm{c}$ & $0,07 \mathbf{a}$ & $6,56 \mathrm{~d}$ \\
\hline \multirow{3}{*}{$\mathrm{T} 4$} & $0-5$ & 0,29 a & $2,7 \mathrm{a}$ & 4,64 a & $2,64 \mathrm{a}$ & $1,62 \mathrm{a}$ & $0,57 \mathrm{a}$ & 0,09 a & $12,6 \mathrm{a}$ \\
\hline & $5-10$ & $0,16 \mathrm{a}$ & $1,52 \mathrm{~b}$ & $2,62 \mathrm{~b}$ & $1,82 \mathrm{~b}$ & $0,84 \mathrm{~b}$ & $0,28 \mathrm{~b}$ & $0,078 \mathrm{a}$ & $8,88 \mathrm{~b}$ \\
\hline & $\begin{array}{l}10-15 \\
15-20\end{array}$ & $\begin{array}{l}0,12 \mathrm{a} \\
0,09 \mathrm{a}\end{array}$ & $\begin{array}{l}0,72 \mathrm{c} \\
0,44 \mathrm{~d}\end{array}$ & $\begin{array}{l}1,24 \mathrm{c} \\
0,76 \mathrm{~d}\end{array}$ & $\begin{array}{l}1,34 \mathrm{c} \\
0,87 \mathrm{~d}\end{array}$ & $\begin{array}{l}0,55 \mathrm{c} \\
0,4 \mathrm{~d}\end{array}$ & $\begin{array}{l}0,15 c \\
0,12 c\end{array}$ & $\begin{array}{l}0,057 \mathrm{~b} \\
0,044 \mathrm{~b}\end{array}$ & $\begin{array}{l}5,92 \mathrm{c} \\
4,24 \mathrm{~d}\end{array}$ \\
\hline \multirow{3}{*}{$\mathrm{T} 5$} & $0-5$ & 0,18 a & 1,9 a & $3,28 \mathbf{a}$ & $2,78 \mathrm{a}$ & $0,6 \mathrm{a}$ & $0,086 \mathrm{a}$ & $0,059 \mathrm{a}$ & $7,52 \mathrm{ab}$ \\
\hline & $\begin{array}{c}5-10 \\
10-15\end{array}$ & $\begin{array}{l}0,13 \text { a } \\
0,11 \text { a }\end{array}$ & $\begin{array}{c}1,25 \mathrm{ab} \\
0,68 \mathrm{~b}\end{array}$ & $\begin{array}{l}2,15 \mathrm{~b} \\
1,17 \mathrm{c}\end{array}$ & $\begin{array}{l}1,67 \mathrm{ab} \\
1,34 \mathrm{ab}\end{array}$ & $\begin{array}{c}0,5 \mathrm{a} \\
0,46 \mathrm{ab}\end{array}$ & $\begin{array}{c}0,058 \mathrm{~b} \\
0,05 \mathrm{~b}\end{array}$ & $\begin{array}{c}0,04 \mathrm{a} \\
0,048 \mathrm{a}\end{array}$ & $\begin{array}{c}8 \mathrm{a} \\
7,52 \mathrm{ab}\end{array}$ \\
\hline & $15-20$ & $0,08 \mathrm{a}$ & $0,54 \mathrm{~b}$ & $0,93 \mathrm{c}$ & $1,02 \mathrm{c}$ & $0,45 \mathrm{ab}$ & $0,042 \mathrm{~b}$ & $0,043 \mathrm{a}$ & $6,32 \mathrm{~b}$ \\
\hline
\end{tabular}


V. T. OUATTARA et al. / Int. J. Biol. Chem. Sci. 12(6): 2901-2921, 2018

Tableau 10: Taux de redistribution verticale dans l'horizon $0-20 \mathrm{~cm}$ de $\mathrm{Mg}, \mathrm{Ca}, \mathrm{K}$ et $\mathrm{Na}$.

\begin{tabular}{|c|c|c|c|c|c|c|c|c|c|c|c|c|c|c|c|c|}
\hline \multirow{2}{*}{ Traitements } & \multicolumn{4}{|l|}{ Mg (p.c.) } & \multicolumn{4}{|c|}{$\mathrm{Ca}$ (p.c.) } & \multicolumn{4}{|c|}{$K(p . c)}$. & \multicolumn{4}{|c|}{$\mathrm{Na}$ (p.c.) } \\
\hline & $0-5$ & $5-10$ & $10-15$ & $15-20$ & $0-5$ & $5-10$ & $10-15$ & $15-20$ & $0-5$ & $5-10$ & $10-15$ & $15-20$ & $0-5$ & $5-10$ & $10-15$ & $15-20$ \\
\hline T0 & $39,58 \mathrm{~d}$ & 25,29 a & $19,77 \mathrm{~b}$ & $15,36 \mathrm{~b}$ & $42,42 \mathrm{a}$ & $24,34 \mathrm{c}$ & $17,38 \mathrm{~b}$ & $15,86 \mathrm{a}$ & 38,74 a & 18,85 a & $18,32 \mathrm{ab}$ & $24,08 \mathrm{a}$ & $25,93 \mathrm{a}$ & $22,84 \mathrm{a}$ & $22,22 \mathrm{a}$ & 29,01 a \\
\hline $\mathrm{Tl}$ & 55,61 a & $21,47 \mathbf{a}$ & $13,90 \mathrm{~d}$ & $9,03 \mathrm{c}$ & $40,98 \mathrm{ab}$ & $30,14 \mathrm{ab}$ & $18,32 \mathrm{~b}$ & $10,55 \mathrm{~b}$ & $40,8 \mathrm{a}$ & $25,92 \mathrm{a}$ & $17,46 \mathrm{ab}$ & $9,81 \mathrm{c}$ & 33,14 a & $26,16 \mathrm{a}$ & $26,16 \mathrm{a}$ & $14,53 \mathrm{~b}$ \\
\hline $\mathrm{T} 2$ & $45,92 \mathrm{bc}$ & $27,56 \mathrm{a}$ & $14,53 \mathrm{~d}$ & $12,07 \mathrm{bc}$ & $31,64 \mathrm{e}$ & $34,86 \mathrm{a}$ & $20,60 \mathrm{a}$ & $15,74 \mathrm{a}$ & $47,56 \mathrm{a}$ & $31,56 \mathrm{a}$ & $12,22 \mathrm{c}$ & $8,81 \mathrm{c}$ & $32,35 \mathrm{a}$ & $30,00 \mathrm{a}$ & $20,00 \mathrm{a}$ & $19,41 \mathrm{ab}$ \\
\hline T3 & $41,25 \mathrm{c}$ & $25,67 \mathrm{a}$ & $18,44 \mathrm{bc}$ & $14,60 \mathrm{~b}$ & $36,44 \mathrm{~d}$ & $29,04 \mathrm{ab}$ & $20,22 \mathrm{a}$ & 14,38 a & $48,85 \mathrm{a}$ & $26,86 \mathrm{a}$ & $14,80 \mathrm{ab}$ & $9,50 \mathrm{c}$ & 24,34 a & 26,59 a & $22,85 \mathrm{a}$ & $25,84 \mathrm{ab}$ \\
\hline T4 & $47,65 \mathrm{~b}$ & $24,52 \mathrm{a}$ & $16,16 \mathrm{~cd}$ & $11,61 \mathrm{bc}$ & $39,27 \mathrm{c}$ & $27,71 \mathrm{c}$ & 20,00 a & $13,01 \mathrm{a}$ & 50,58 a & $25,07 \mathrm{a}$ & $13,73 \mathrm{c}$ & $10,63 \mathrm{c}$ & $33,46 \mathrm{a}$ & $29,00 \mathrm{a}$ & 21,19 a & $16,36 \mathrm{ab}$ \\
\hline T5 & $29,69 \mathrm{e}$ & $25,11 \mathrm{a}$ & 22,82 a & $22,38 \mathrm{a}$ & $40,31 \mathrm{c}$ & $24,70 \mathrm{c}$ & 19,91 a & $15,08 \mathrm{a}$ & $36,44 \mathrm{a}$ & 24,58 a & 21,19 a & $17,80 \mathrm{~b}$ & $31,05 \mathrm{a}$ & $21,05 \mathrm{a}$ & $25,26 \mathrm{a}$ & $22,63 \mathrm{ab}$ \\
\hline Moyenne & 43,28 & 24,94 & 17,600 & 14,170 & 38,51 & 28,46 & 19,410 & 14,100 & 44,83 & 25,47 & 16,290 & 13,440 & 30,05 & 25,94 & 22,950 & 21,300 \\
\hline $\mathrm{CV}$ & 2,3 & 3,60 & 2,700 & 4,000 & 1,8 & 3,30 & 1,300 & 3,600 & 6,2 & 5,70 & 5,500 & 6,200 & 7 & 9,30 & 6,700 & 8,100 \\
\hline Probabilité & $<0,001$ & 0,16 & $<0,001$ & $<0,001$ & $<0,001$ & 0,012 & 0,005 & 0,012 & 0,184 & 0,47 & 0,022 & $<0,001$ & 0,255 & 0,45 & 0,436 & 0,035 \\
\hline
\end{tabular}


Tableau 11 : Taux de redistribution verticale dans l'horizon $0-20 \mathrm{~cm}$ de la CEC, N, C et SB.

\begin{tabular}{|c|c|c|c|c|c|c|c|c|c|c|c|c|c|c|c|c|}
\hline \multirow{2}{*}{ Traitements } & \multicolumn{4}{|c|}{$\mathrm{CEC}$ (p.c.) } & \multicolumn{4}{|c|}{$\mathbf{N}$ (p.c.) } & \multicolumn{4}{|c|}{$\mathrm{C}$ (p.c.) } & \multicolumn{4}{|c|}{ SB (p.c.) } \\
\hline & $0-5$ & $5-10$ & $10-15$ & $15-20$ & $0-5$ & $5-10$ & $10-15$ & $15-20$ & 0.5 & $5-10$ & $10-15$ & $15-20$ & 0.5 & $5-10$ & $10-15$ & $15-20$ \\
\hline T0 & $34,76 \mathrm{a}$ & $25,67 \mathrm{a}$ & $19,25 \mathrm{c}$ & $20,32 \mathrm{a}$ & $36,42 \mathrm{a}$ & 24,83 a & 19,87 a & $18,21 \mathrm{a}$ & $43,18 \mathrm{a}$ & $27,86 \mathrm{a}$ & 20,61 a & 13,93 a & 41,87 a & $24,43 \mathrm{~b}$ & $17,83 \mathrm{bc}$ & $15,90 \mathrm{a}$ \\
\hline $\mathrm{Tl}$ & $32,24 \mathrm{a}$ & $28,57 \mathrm{a}$ & $21,03 \mathrm{~b}$ & $18,16 \mathrm{~b}$ & $39,02 \mathrm{a}$ & 25,61 a & 19,51 a & $16,46 \mathrm{a}$ & $46,38 \mathrm{a}$ & 25,94 a & $17,30 \mathrm{a}$ & $10,38 \mathrm{ab}$ & $45,15 \mathrm{a}$ & $27,64 \mathrm{~b}$ & $17,13 \mathrm{c}$ & $10,11 \mathrm{c}$ \\
\hline $\mathrm{T} 2$ & 39,68 a & 29,88 a & $16,06 \mathrm{~d}$ & $14,38 \mathrm{c}$ & $39,53 \mathrm{a}$ & 27,91 a & 19,77 a & $12,79 \mathrm{a}$ & 45,32 a & 29,64 a & 7,27 a & $10,65 \mathrm{ab}$ & $37,65 \mathrm{~b}$ & $32,25 \mathrm{a}$ & $17,85 \mathrm{bc}$ & $13,88 \mathrm{ab}$ \\
\hline $\mathrm{T} 3$ & 36,68 a & $21,40 \mathrm{~b}$ & $23,83 \mathrm{a}$ & $18,09 \mathrm{~b}$ & $42,03 \mathrm{a}$ & 24,64 a & 17,39 a & 15,94 a & $53,54 \mathrm{a}$ & 23,77 a & $13,07 \mathbf{a}$ & $9,44 \mathrm{ab}$ & $39,04 \mathrm{~b}$ & $27,91 \mathrm{~b}$ & $19,17 \mathrm{~b}$ & $13,91 \mathrm{ab}$ \\
\hline T4 & $39,82 \mathrm{a}$ & 28,07 a & $18,71 \mathrm{c}$ & $13,40 \mathrm{~d}$ & $43,61 \mathrm{a}$ & 24,06 a & $18,05 \mathrm{a}$ & $14,29 \mathrm{a}$ & 53,68 a & $30,32 \mathrm{a}$ & 14,31 a & $8,75 \mathrm{~b}$ & $42,94 \mathrm{a}$ & $26,47 \mathrm{~b}$ & $18,21 \mathrm{bc}$ & $12,34 \mathrm{~b}$ \\
\hline T5 & $26,8 \mathrm{~b}$ & $26,39 \mathrm{a}$ & $24,90 \mathrm{a}$ & 21,91 a & $26 a$ & $26,00 \mathrm{a}$ & $22,0 \mathrm{a}$ & $16,00 \mathrm{a}$ & $45,05 \mathrm{a}$ & 29,48 a & 16,04 a & $12,74 \mathrm{ab}$ & $37,83 \mathrm{~b}$ & $24,79 \mathrm{~b}$ & $20,60 \mathrm{a}$ & $16,78 \mathrm{a}$ \\
\hline Moyenne & 34,67 & 26,66 & 20,61 & 17,53 & 37,77 & 25,51 & 19,43 & 15,62 & 47,86 & 27,84 & 14,770 & 10,980 & 40,75 & 27,25 & 18,46 & 13,82 \\
\hline $\mathrm{CV}$ & 3,5 & 2,00 & 1,30 & 1,00 & 15,4 & 14,10 & 9,70 & 5,90 & 4 & 8,40 & 17,200 & 5,400 & 1,4 & 3,00 & 1,30 & 3,20 \\
\hline Probabilité & 0,005 & 0,002 & $=0,001$ & $=0,001$ & 0,694 & 0,99 & 0,860 & 0,219 & 0,129 & 0,735 & 0,176 & 0,037 & 0,003 & 0,029 & 0,004 & 0,003 \\
\hline
\end{tabular}




\section{DISCUSSION}

Effets des régimes de fertilisation sur la matière organique et le complexe adsorbant du sol

Le régime de fertilisation composé de compost $+184 \mathrm{~kg} /$ ha de $\mathrm{P}_{2} \mathrm{O}_{5}$ a fourni non seulement les meilleurs résultats avec 5,42 p.c. du taux de matière organique, mais aussi, a été meilleur pour l'ensemble des paramètres, cations $\left(\mathrm{Mg}^{2+}\right.$ et $\left.\mathrm{K}^{+}\right)$et le complexe d'échange cationique (CEC) tout en gardant un niveau acceptable de $\mathrm{Ca}^{2+}$.

Cela est dû au fait que le compost aurait eu ici pour rôle essentiel d'apporter du potassium, du magnésium et d'élever le taux de matière organique du sol. En effet, l'engrais, à travers les anions phosphates libérés et de sa teneur élevée en calcium, a eu l'effet d'augmenter la capacité d'échange cationique des sols. Des résultats similaires ont été observés par d'autres auteurs (N'guessan et al., 2016).

\section{Effets des différents régimes de fertilisation sur les équilibres chimiques des sols}

L'analyse des équilibres chimiques a montré un pourcentage de calcium élevé dans le sol de la jachère et l'engrais minéral seul, mais les pourcentages en magnésium et en potassium y sont les plus faibles contrairement aux traitements organominéraux et organiques.

Ces résultats pourraient s'expliquer par la nature du sol du site d'expérimentation qui contiendrait suffisamment de calcium et du TSP qui renferme en son sein du calcium. Cela a été montré par N'guessan et al. (2016). Par contre, le compost contient une teneur importante en potassium et relativement élevée en magnésium comme l'ont montré Adejobi et al. (2014). Le sol ayant reçu du compost semble avoir les teneurs en bases équilibrées $(70$ p.c. calcium, 24 p.c. magnésium, et 6 p.c. potassium) par rapport aux autres traitements. En effet, Snoeck et al. (2006) préconise 68 p.c. de calcium, 24 p.c. de magnésium et 8 p.c. de potassium comme optimum des équilibres $\mathrm{Ca}-\mathrm{Mg}-\mathrm{K}$ dans les sols sous cacaoyers. Les régimes de fertilisation organo-phosphatés ont eu un équilibre $\mathrm{Ca}-\mathrm{Mg}-\mathrm{K}$ en faveur du magnésium et surtout du potassium. Cette situation semble être influencée par la matière organique. Concernant les traitements témoin et engrais minéral phosphaté seul, l'équilibre a été plus favorable au calcium. Les régimes de fertilisation organo-phosphatés et organique (avec un rapport d'environ 3) ont donné un rapport $\mathrm{K} / \mathrm{CEC}$ supérieur à 1,5 qui est l'optimum. Cela dénote d'une teneur en potassium élevée dans ces sols fertilisés. Pour le traitement de fertilisation minérale et le témoin absolu, un faible rapport (respectivement 0,78 et 0,64 ) indique une déficience en potassium qui sont consommé et non renouvelé car le TSP ne contient pas de potassium.

Le rapport $\mathrm{N} / \mathrm{K}$ a été proche de l'optimum qui est de 0,75 , pour les traitements organo-phosphatés et organique. Cependant, le compost $+184 \mathrm{~kg} / \mathrm{ha} \mathrm{P}_{2} \mathrm{O}_{5}$ avec un rapport $\mathrm{N} / \mathrm{K}$ de 0,76 semble plus rapproché de cet optimum. Cela suggère un bon équilibre entre le phosphore et le potassium. Toutefois, les traitements compost, compost $+122,6 \mathrm{~kg} / \mathrm{ha}$ $\mathrm{P}_{2} \mathrm{O}_{5}$ et compost $+92 \mathrm{~kg} / \mathrm{ha} \quad \mathrm{P}_{2} \mathrm{O}_{5}$ avec respectivement des rapports $\mathrm{N} / \mathrm{K}$ de $0,84,0,68$ et 0,66 conservent un équilibre assez bon. Par contre, le témoin sans fertilisation et l'engrais minéral phosphaté ont un rapport élevé qui pourrait être dû à une déficience en azote plus importante qu'en potassium.

\section{Effets des régimes de fertilisation sur la rédistribution des éléments nutritifs dans la couche $0-20 \mathrm{~cm}$}

Les éléments minéraux ont été accumulés en surface (horizon 0-10 cm) par application des traitements organo-minéraux et organique seul. La répartition a été plus homogène pour T5. Comme l'ont montré Ouattara et al. (2017), la matière organique retient les éléments nutritifs en surface alors que l'engrais minéral seul accélère leur migration verticale. Aussi avons-nous constaté que dans l'ensemble des horizons étudiés, les teneurs baissent de la surface vers la profondeur. En effet selon Ouattara et al. (2017), les sols contenant du compost retiennent les éléments nutritifs en surface. D'autres auteurs indiquent qu'en présence de matière organique, les nutriments sont disponibles pour les plantes (Adekiya et al., 2015). Par ailleurs, ces résultats montrent 
aussi que la matière organique a été minéralisée beaucoup plus en présence des doses d'engrais. Et la minéralisation est d'autant plus importante que la dose est faible comme c'est le cas de $\mathrm{T} 4$ et $\mathrm{T} 3$. Ces résultats sont similaires à ceux de Plassard et al. (2015). Fontaine et Barot (2005) ont montré dans leur étude qu'un apport de matière organique peut augmenter la minéralisation des matières organiques du sol de 12 à 400 p.c. en fonction de différents discriminants liés à la nature du sol, du substrat qui le génère et à la composition communautaire microbienne. Falinirina (2010) à Madagascar pour sa part, a constaté que quelle que soit la nature et la dose d'apport organique, l'apport de fertilisants minéraux augmente la vitesse de croissance de maïs. En effet, on admet que de façon générale, qu'un gramme de matière organique contribue à 5 fois plus à la CEC d'un sol qu'un gramme d'argile (Bigorre, 2000. Pour les sols très acides en présence d'aluminium soluble, le magnésium peut être insolubilisé par précipitation avec les composés d'aluminium. Il est libéré dès que le pHeau remonte suite à un chaulage (Schvartz, 2005) ou un apport de matière organique. Les concentrations en carbone et en azote organique dans le système de conservation comme c'est le cas de notre parcelle d'expérimentation sont en général supérieures dans les 10 premiers $\mathrm{cm}$ du sol par rapport à un sol labouré et décroissent fortement dans les horizons sous-jacents. Ces résultats sont similaires à ceux de D'Haene et al. (2008). La baisse de stocks de carbone en profondeur est liée au système racinaire des cultures qui est plus dense en surface (Munoz et Beer, 2001). En effet, ces auteurs dans leurs études fines de la dynamique verticale du sytème racinaire du cacaoyer ont montré que plus de 90 p.c. des racines fines est situé dans l'horizon $0-10 \mathrm{~cm}$ mais surtout dans celui $0-5 \mathrm{~cm}$. Aussi, d'autres auteurs ont montré que dans les systèmes de conservation, les racines fines sont à la surface contrairement aux sols labourés, d'où une diminution de la rhizodéposition en profondeur et une baisse des entrées de carbone (Jourdan, 2014).

L'accumulation des éléments nutritifs est également modifiée par les techniques de travail du sol. Ainsi, les micronutriments présentent une stratification verticale identique à celle des pools de carbone, azote, phosphore, zinc et potassium s'accumulent en surface dans les systèmes de conservation et diminuent en profondeur tandis qu'ils sont repartis de façon homogène sur la couche de sol labourée (Wright et al., 2007). Dans la même veine, Messiga et al. (2012) montrent que le phosphore est reparti en surface dans les sols non labourés comme c'est le cas dans notre étude. Cest une bonne pratique dans les sols sous cacaoyer dont les racines fines, pool de l'absorption des nutriments, sont distribuées dans la couche 0-10 cm (Munoz et Beer, 2001). De plus, le compost est pourvoyeur d'éléments nutritif comme l'ont montré Adden (2017) dans ses travaux sur la productivité des vergers cacaoyers au togo. Soma (2010) étudiant l'effet des amendements organiques dans un sol ferrugineux tropical a également noté l'importance de la matière organique pour les plantes dans ce sol.

\section{Conclusion}

L'expérimentation menée en station de recherche à Divo (Côte d'Ivoire) en vue d'étudier les effets de la fertilisation organophosphatée sur la dynamique verticale dans 1'horizon 0-20 cm, du complexe adsorbant, de la matière organique et de quelques équilibres chimiques dans les sols sous cacaoyers a montré que la fertilisation mixte était meilleure que la fertilisation organique seule et minérale seule vis-à-vis du complexe, de la matière organique et évidemment des équilibres chimiques. Le traitement T2 s'est révélé meilleur mais aussi T3, T4 et parfois T1 ont amélioré les caractéristiques étudiées. La matière organique tout en se minéralisant a aussi permis l'accumulation à travers le taux de redistribution des éléments nutritifs en surface surtout pour les régimes de fertilisation mixte, pool de l'absorption racinaire chez le cacaoyer.

\section{CONTRIBUTIONS DES AUTEURS}

Ce travail a été réalisé avec la contribution des personnes suivantes: VTO, ZK et GFM ont participé à la conception, à l'élaboration et à la correction du manuscrit ; EKK, MGT, LAK et BK ont assuré la supervision et la conduite des travaux sur le 
terrain, la rédaction et la mise en forme de l'article ainsi que les analyses statistiques des données; $\mathrm{EAD}$ et $\mathrm{MC}$ ont supervisé la conduite des travaux, la conception, l'élaboration et la rédaction du manuscrit.

\section{REMERCIEMENTS}

Cette étude a été réalisée dans le cadre du Projet initié entre le Centre National de Recherche Agronomique (CNRA) et l'Office Chérifien du Phosphate (OCP). Les auteurs remercient le CNRA, l'OCP, LDC, ICRAF, FIS ainsi que l'Université Félix HouphouëtBoigny et l'Institut National Polytechnique Houphouët-Boigny de Yamoussoukro pour leur soutien financier et matériel.

\section{RÉFÉRENCES}

Aguilar P, Paulin D, Nkamleu G, Raillard A, Deheuvels O, Petithuguenin P. 2005. L'évolution des vergers de cacaoyers en Côte d'Ivoire entre 1995 et 2002. In: Cocoa Producers, Alliance L (ed) 14th International Cocoa Research Conference, Accra, Ghana, 1167-1175.

Adejobi KB, Akanbi OS, Ugioro O, Adeosun SA, Mohammed I, Nduka BA, Adeniyi DO. 2014. Comparative effects of NPK fertilizer, cowpea pod husk and some tree crops wastes on soil, leaf chemical properties and growth performance of cocoa (Theobroma cacao L.). AJPS, 8(2): 103-107

Adden KA. 2017. Amélioration de la productivité des vergers de cacaoyers (Theobroma cacao Linn.) pour une gestion forestière durable au Togo. Thèse de l'Université de Lomé (Togo), Ecole Superieure d'Agronomie, spécialité Science des Agroressources p 138 https://hal.archives-ouvertes.fr/tel01567889

Adekiya AO, Agbede TM, Ojeniyi SO. 2015. The effect of three years of tillage and poultry manure application on soil and plant nutrient composition, growth and yield of cocoyam. Expl Agric., 52(3): 466-476.

DOI:10.1017/S0014479715000253

Assiri AA, Konan A, N'Guessan KF, Kébé BI, Kassin KE, Couloud JY, Yapo AR, Yoro GR, Yao-Kouamé A. 2015.
Comparaison de deux techniques de replantation cacaoyère sur antécédents culturaux non-forestiers en Côte D'Ivoire. ACSJ, 23(4): 365 - 378.

Bigorre F. 2000. Influence de la pédogenèse et de l'usage des sols sur leurs propriétés physiques. Mécanismes d'évolution et éléments de prévision. Thèse de l'Université Henri Poincaré Nancy I (France) G.F.D. Sciences de la terre, spécialité Science du sol p 148

D'Haene K, Vermang J, Cornelis WM, Leroy BLM, Schiettecatte W, De Neve S, Gabriels D, Hofman G. 2008 Reduced tillage effects on physical properties of silt loam soils growing root crops. Soil Tillage Res., 99:279-290.

Falinirina MV. 2010. Valorisation agricole des apports organiques contenus dans les déchets urbains: qualité des matières organiques et service écosystémique. Thèse de doctorat, Ecole Supérieure des Sciences Agronomiques d'Antananarivo, Université d'Antananarivo.p 192 .

Fontaine S, Barot S. 2005. Size and functional diversity of microbe populations control plant persistence and long-term soil carbon accumulation. Ecology Letters. 8(10): $\quad 1075-1087 . \quad$ DOI: http://dx.doi.org/10.1111/j.14610248.2005.00813.x

Gockowski J, Sonwa DJ. 2010. Cocoa Intensification Scenarios and Their Predicted Impact on $\mathrm{CO}_{2}$ Emissions, Biodiversity Conservation, and Rural Livelihoods in the Guinea Rain Forest of West Africa. Environnemental Management, 48(2): 307-321. DOI : http://dx.doi.org/10.1007/s00267-0109602-3

Hartemink AE. 2005. Nutrient stocks, nutrient cycling and soil changes in cocoa ecosystems: A review. Advances in Agronomy, 8 : 227-253.

Hegman W, Wang D, Borer C. 1999. Estimation of lake champlain Basinwide Nonpoint Source Phosphorus Export. Technical report No 31. Lake Champlain Basin Program. Grand Isle, Vermont, Etats-Unis p 69. 
Jackson ML. 1958. Soil chemical analysis. ASA Monograph, $\mathrm{N}^{0} 9$ part 2- Methods of soil analysis.

Jourdan C. 2014. Caractériser, comprendre et modéliser l'architecture, le développement et le fonctionnement des systèmes racinaires des arbres tropicaux plantés - Rôle des racines profondes. Mémoire présenté en vue d'obtenir L'Habilitation à Diriger des Recherches, Ecole doctorale Systèmes Intégrés en Biologie, Agronomie, Géosciences, Hydrosciences, Environnement (SIBAGHE). Université de Montpellier II (France) p 97

Kassin KE. 2009. Etudes de conditions pédoclimatiques pour la replantation cacaoyère au centre-ouest de la Côte d'Ivoire: cas des départements de Divo et Gagnoa. Thèse de Doctorat unique, UFR STRM, Université de Cocody, Abidjan, Côte d'Ivoire, p 112

Kébé BI, N'Guessan KF, Tahi GM, Assiri AA, Koko LK, Kohi NJ, Irié Bi Z, Koffi N. 2009. Bien cultiver le cacaoyer en Côte d'Ivoire. CTA, Direction des programmes de recherche et de l'appui au développement - Direction des Innovations et systèmes d'information, CNRA, Abidjan, Côte d'Ivoire, $\mathrm{p} 4$.

KOKO L. 2013. Bien faire du « compost enrichi » à base de résidus d'écabossage en 3 mois. Fiche technique illustré. Projet régional WCF Cocoa Livelihoods Program Challenge Grant/CNRA Côte d'Ivoire. $23 \mathrm{p}$.

Koko LK, Kassin KE, Yoro G, NGoran K, Yao-Kouamé A. 2009. Corrélations entre le vieillissement précoce des cacaoyers et les caractéristiques morphopédologiques dans le Sud-Ouest de la Côte d'Ivoire. J. Appl. Biosci., 24: 1508-1519.

Konaté Z, Assiri AA, Messoum FG, Sekou A, Camara M, Yao-Kouamé A. 2016. Identifcation de quelques contraintes paysannes en replantation cacaoyère en Côte d'Ivoire. Science de la vie, de la terre et agronomie. Revue CAMES - 04(2): 51-57.
Letkeman LP, Tiessen H, Campbell CA. 1996. Phosphorus transformations and redistribution during pedogenesis of western Canadian soils. Geoderma, 71: 201-218.

Messiga JA, Ziadi N, Morel C, Grant C, Tremblay Gd, Lamarre G, Parent L-E. 2012. Long term impact of tillage practices and biennial $\mathrm{P}$ and $\mathrm{N}$ fertilization on maize and soybean yields and soil $\mathrm{P}$ status. Field Crops Research. $133: 10-22$. DOI:10.1016/j.fcr.2012.03.009

Munoz F, Beer J. 2001. Fine root dynamics of shade cacao plantation in Costa rica. Agroforestery Systems, 54:119-130.

N'guessan KJ-C, Akotto OF, Snoeck D, Camara M, Yao - Kouamé A. 2016. Potentiel de fertilité chimique des vergers de cacaoyer Theobroma cacao $\mathrm{L}$. (Malvacea) en Côte d'Ivoire .IJIAS, 18(3) : 868-879.

Ouattara TV, Kassin KE, Koko LJ, Tahi GM, Assi ME, Amari G, Dick E, Camara M. 2017. Effets de la fertilisation organophosphatée sur la biodisponibilité du phosphore, la teneur en aluminium et le ph des sols sous cacaoyers dans la région de Divo en Côte d'ivoire. J. Appl. Biosci., 118: 11754-11767. DOI : http://dx.doi.org/10.4314/jab.v118i1.2.

Plassard C, Robin A, Le Cadre E, Marsden C, Trap J, Herrmann L, Waithaisong K, Lesueur D, Blanchart E, Chapuis-Lardy L, Hinsinger P. 2015. Améliorer la biodisponibilité du phosphore : comment valoriser les compétences des plantes et les mécanismes biologiques du sol ? Innovations Agronomiques, 43 : 115-138

Pleysier JL, Juo ASR, Herbillon A. 1979. Ion exchange equilibria involving $\mathrm{Al}$ in a kaolinitic Ultisol. SSSAJ, 43(5):875-880. DOI: https://dx.doi.org/10.2136/sssaj197 9.03615995004300050011x

Sanni LA, Ugoso ES, Faborode MO. 2015. Effect of dryer parameters on the drying characteristics and quality of cassava flour. AJFST, 6(7): 185-193. 
Schvartz C, Muller J-C, Decroux J. 2005. Guide de la fertilisation raisonnée, Grandes cultures et prairies, Paris : COMIFER-Éditions France Agricole, $p$ 414 ,

Snoeck D, Abekoe MK, Afrifa AA, Appiah MRK. 2006. "The soil diagnostic method to compute fertilizer requirements in cocoa plantations", Proc, Int, Conf, on Soil Science, Accra, Ghana, 16-21 July 2006, $10 \mathrm{p}$,

Soma DM. 2010. Effet des apports répétés de diverses sources d'amendements organiques dans un sol ferrugineux tropical lessivé (Saria, Burkina Faso) sur la biodisponibilité du phosphore et la production du sorgho, Diplôme d'Etudes Approfondies, Université polytechnique de Bobo-Dioulasso (Burkina Faso), p 47
Stadelmann P, Lovas R, Butscher E. 2002. 20 Jahre Sanierung und Überwachung des Baldeggersees. Mitteilungen der naturforschenden Gesellschaft Luzern, 37: 115-164.

Wright SJ, Kathryn ES, Beckman N, Corlett RT, Rodolfo D, Muller-Landau HC, GNI, Peres CA, Wang BC. 2007. The Plight of Large Animals in Tropical Forests and the Consequences for Plant Regeneration, Biotropica, 39(3):289291,

Yoro GR. 2004. Reconnaissance des sols favorables aux cacaoyers, Cours de formation des producteurs de cacao et des agents ANADER détachés auprès de STCP, Rapport technique, CNRA, p 8. 